\title{
The weak extension property and finite axiomatizability for quasivarieties
}

\author{
by \\ Wiesław Dziobiak (Mayagüez, PR), Miklós Maróti (Szeged), \\ Ralph McKenzie (Nashville, TN), and Anvar Nurakunov (Bishkek)
}

\begin{abstract}
We define and compare a selection of congruence properties of quasivarieties, including the relative congruence meet semi-distributivity, $\operatorname{RSD}(\wedge)$, and the weak extension property, WEP. We prove that if $\mathcal{K} \subseteq \mathcal{L} \subseteq \mathcal{L}^{\prime}$ are quasivarieties of finite signature, and $\mathcal{L}^{\prime}$ is finitely generated while $\mathcal{K}=\mathrm{WEP}$, then $\mathcal{K}$ is finitely axiomatizable relative to $\mathcal{L}$. We prove for any quasivariety $\mathcal{K}$ that $\mathcal{K}=\operatorname{RSD}(\wedge)$ iff $\mathcal{K}$ has pseudo-complemented congruence lattices and $\mathcal{K} \mid=$ WEP. Applying these results and other results proved by M. Maróti and R. McKenzie [Studia Logica 78 (2004)] we prove that a finitely generated quasivariety $\mathcal{L}$ of finite signature is finitely axiomatizable provided that $\mathcal{L}$ satisfies $\operatorname{RSD}(\wedge)$, or that $\mathcal{L}$ is relatively congruence modular and is included in a residually small congruence modular variety. This yields as a corollary the full version of R. Willard's theorem for quasivarieties and partially proves a conjecture of D. Pigozzi. Finally, we provide a quasi-Maltsev type characterization for $\operatorname{RSD}(\wedge)$ quasivarieties and supply an algorithm for recognizing when the quasivariety generated by a finite set of finite algebras satisfies $\operatorname{RSD}(\wedge)$.
\end{abstract}

1. Introduction. For more information on the basic notions of general algebra introduced below and used throughout this paper, we refer the reader to [22]. By an algebra we shall mean a system of the form $\mathbf{A}=\left\langle A, F_{t}^{\mathbf{A}}\right.$ $(t \in T)\rangle$ where $A$ is a non-void set, $T$ is a set, and for $t \in T, F_{t}^{\mathbf{A}}$ is a $\sigma(t)$-ary operation over $A$ for some non-negative integer $\sigma(t)$. The function $\sigma: T \rightarrow \omega$ giving the arities of the operations is the signature of $\mathbf{A}$. Such a signature $\sigma$ determines, of course, a first-order language $L_{\sigma}$ with operation symbols but no relation symbols. An algebra of signature $\sigma$ is nothing other than a model of the language $L_{\sigma}$. Algebras with the same signature are said to be similar. A signature $\sigma: T \rightarrow \omega$ is finite just in case $T$ is a finite set.

We interpret the class-operators $\mathrm{H}, \mathrm{S}, \mathrm{P}$ in the inclusive sense, so that if $\mathcal{K}$ is a class of similar algebras, then $\mathrm{S}(\mathcal{K})$ denotes the class of all algebras isomorphic to a subalgebra of some algebra in $\mathcal{K}, \mathrm{H}(\mathcal{K})$ denotes the class of

2000 Mathematics Subject Classification: 08A99, 08B10, 03C05, 08C15.

Key words and phrases: quasivariety, finite axiomatizability, congruence properties. 
all surjective homomorphic images of algebras in $\mathcal{K}$, and $\mathrm{P}(\mathcal{K})$ denotes the class of all algebras isomorphic to a Cartesian product of a system of algebras from $\mathcal{K}$. We use $\mathrm{P}_{u}(\mathcal{K})$ to denote the class of all algebras isomorphic to an ultraproduct of a system of algebras from $\mathcal{K}$, and $\mathrm{P}_{r}(\mathcal{K})$ to denote the class of all algebras isomorphic to a reduced product of a system of algebras from $\mathcal{K}$.

A quasivariety is a class $\mathcal{K}$ of similar algebras such that $\mathrm{S}(\mathcal{K})=\mathrm{P}_{r}(\mathcal{K})$ $=\mathcal{K}$. By a classical result of A. I. Maltsev [16], a quasivariety is the same thing as a class of similar algebras axiomatized by a set of quasi-equations of the corresponding first order language. By quasi-equation we understand a sentence of the form

$$
(\forall \bar{x})\left(\left\{s_{1}(\bar{x}) \approx t_{1}(\bar{x}) \wedge \cdots \wedge s_{n}(\bar{x}) \approx t_{n}(\bar{x})\right\} \rightarrow s(\bar{x}) \approx t(\bar{x})\right)
$$

where $s_{i}, s, t_{j}, t$ are terms. According to results of G. Grätzer and H. Lakser [10], for a class $\mathcal{K}$ of similar algebras, $\mathrm{SPP}_{u}(\mathcal{K})=\mathrm{SP}_{r}(\mathcal{K})$ is the smallest quasivariety containing $\mathcal{K}$; and $\mathcal{K}$ is a quasivariety iff $\mathrm{S}(\mathcal{K})=\mathrm{P}(\mathcal{K})=\mathrm{P}_{u}(\mathcal{K})$ $=\mathcal{K}$. Since $\mathrm{P}(\mathcal{K})$ contains a product of an empty system of algebras, a quasivariety contains the one-element algebras of its signature.

A variety is a class $\mathcal{K}$ of algebras of the same signature such that $\mathrm{H}(\mathcal{K})=$ $\mathrm{S}(\mathcal{K})=\mathrm{P}(\mathcal{K})=\mathcal{K}$. According to a famous theorem of G. Birkhoff [4], a variety is the same thing as a class of similar algebras axiomatized by a set of equations of the corresponding first order language, where by equation we mean a sentence of the form $(\forall \bar{x})(s(\bar{x}) \approx t(\bar{x}))$ for some terms $s(\bar{x})$ and $t(\bar{x})$. Varieties are quasivarieties; in fact, they are precisely the quasivarieties $\mathcal{K}$ that satisfy $\mathrm{H}(\mathcal{K})=\mathcal{K}$. The smallest variety containing a class $\mathcal{K}$ of similar algebras is the class $\operatorname{HSP}(\mathcal{K})$.

A quasivariety $\mathcal{K}$ is said to be finitely generated if $\mathcal{K}=\operatorname{SPP}_{u}(\mathcal{M})$ for some finite set $\mathcal{M}$ of finite algebras. In this case, of course, $\mathcal{K}=\operatorname{SP}(\mathcal{M})$. Likewise, a variety $\mathcal{K}$ is said to be finitely generated (i.e., as a variety) if $\mathcal{K}=\operatorname{HSP}(\mathcal{M})$ for some finite set $\mathcal{M}$ of finite algebras.

For a quasivariety $\mathcal{K}$ and $\mathbf{A} \in \mathcal{K}$ we write $\mathbf{C o n} \mathbf{A}$ for the lattice of congruences of $\mathbf{A}$, and $\mathbf{C o n}_{\mathcal{K}} \mathbf{A}$ for the lattice of $\mathcal{K}$-congruences of $\mathbf{A}$, where a $\mathcal{K}$-congruence is any congruence $\theta$ such that $\mathbf{A} / \theta \in \mathcal{K}$. Recall that the join $\checkmark$ in $\mathbf{C o n} \mathbf{A}$ and the join $\vee^{\mathcal{K}}$ in $\operatorname{Con}_{\mathcal{K}} \mathbf{A}$ are related by the equation

$$
\alpha \vee^{\mathcal{K}} \beta=\overline{\alpha \vee \beta} \mathcal{K} \quad \text { for }\{\alpha, \beta\} \subseteq \operatorname{Con}_{\mathcal{K}} \mathbf{A}
$$

where for any $\theta \in \operatorname{Con} \mathbf{A}, \bar{\theta}^{\mathcal{K}}$ is the smallest member of $\operatorname{Con}_{\mathcal{K}} \mathbf{A}$ containing $\theta$.

This paper is devoted to the study of a collection of sufficient conditions for finite axiomatizability of a finitely generated variety or quasivariety. All such conditions we encounter will be expressed as combinations of properties of the lattice of congruences, or the lattice of relative congruences (or a combination of the two) holding for all algebras in a quasivariety. We now define the most central congruence properties we shall use. Recall the following 
lattice formulas:

$$
\begin{array}{rlrl}
x \wedge(y \vee z) & =(x \wedge y) \vee(x \wedge z) & & \text { (distributive law) } \\
x \geq y \rightarrow x \wedge(y \vee z) & =(x \wedge y) \vee(x \wedge z) & & \text { (modular law), } \\
x \wedge y=x \wedge z \rightarrow x \wedge(y \vee z) & =(x \wedge y) \vee(x \wedge z) & & \text { (meet semi-distributive } \\
& & \text { law). }
\end{array}
$$

A lattice $\mathbf{L}$ is distributive (respectively modular, or meet semi-distributive) if the distributive law (respectively the modular law or the meet semidistributive law) holds for all elements $x, y, z$ in $\mathbf{L}$.

A quasivariety is said to satisfy $\mathrm{CM}$ (respectively, $\mathrm{CD}$ or $\mathrm{SD}(\wedge)$ ) if and only if all the lattices $\mathbf{C o n} \mathbf{A}$ with $\mathbf{A} \in \mathcal{K}$ are modular lattices (respectively, distributive lattices, or meet semi-distributive lattices). A quasivariety is said to satisfy $\operatorname{RCM}$ (respectively, $\operatorname{RCD}$ or $\operatorname{RSD}(\wedge)$ ) if and only if all the lattices $\mathbf{C o n}_{\mathcal{K}} \mathbf{A}$ with $\mathbf{A} \in \mathcal{K}$ are modular lattices (respectively, distributive lattices, or meet semi-distributive lattices).

The serious study of the finite axiomatizability property in finitely generated varieties originated with Roger Lyndon in the 1950's. A decade earlier, Garrett Birkhoff had asked whether the equational theory of a finite algebra of finite signature must be finitely axiomatizable. Lyndon proved in [14] that the equational theory of every two-element algebra of finite signature is finitely axiomatizable and also provided in [15] the first example of a finite algebra (a 7-element groupoid) the equational theory of which is not finitely axiomatizable. In the half-century since these papers appeared, many interesting and important results concerning the finite or non-finite axiomatizability of finitely generated varieties have been established. (See [17] for a brief account of the results obtained.) The following three results have been regarded with respect to the range of their applicability as the most distinguished positive results in this literature.

BAKeR's TheOREM (K. Baker [2]). Let $\mathcal{K}$ be a variety of finite signature. If $\mathcal{K}$ is congruence distributive and finitely generated, then $\mathcal{K}$ is finitely axiomatizable.

McKenzIE's Theorem (R. McKenzie [19]). Let $\mathcal{K}$ be a variety of finite signature. If $\mathcal{K}$ is congruence modular, residually small, and finitely generated, then $\mathcal{K}$ is finitely axiomatizable.

Willard's TheOREm (R. Willard [27]). Let $\mathcal{K}$ be a variety of finite signature. If $\mathcal{K}$ is congruence meet semi-distributive and has a finite residual bound, then $\mathcal{K}$ is finitely axiomatizable.

Once each of the above theorems had been proved, it prompted attempts to find its valid extensions within finitely generated quasivarieties, or equivalently speaking, within quasi-equational classes generated by finite sets of 
finite algebras (see for example W. J. Blok and D. Pigozzi [5], J. Czelakowski and W. Dziobiak [6], W. Dziobiak [7], K. Kearnes and R. McKenzie [13], M. Maróti and R. McKenzie [17], A. Nurakunov [23], [24], and D. Pigozzi [25]). A quasi-equational class is a universal Horn class which contains a oneelement algebra.

We extend Willard's theorem to quasivarieties. Our extension is based on earlier work of M. Maróti and R. McKenzie [17]. It relies on the weak extension property, which is introduced and developed in Section 2 below.

In [25], D. Pigozzi proved the following theorem: If $\mathcal{K}$ is a finitely generated and relatively congruence distributive quasivariety of finite signature, then $\mathcal{K}$ is finitely axiomatizable. D. Pigozzi's theorem fully extended K. Baker's theorem to quasivarieties, and is itself extended by the new results mentioned above. Having proved his theorem and looked for a common extension into quasivarieties of both his and R. McKenzie's theorem, D. Pigozzi made the following conjecture in the late 1980's.

PigOzZI's CONJECTURE. If $\mathcal{K}$ is a finitely generated and relatively congruence modular quasivariety of finite signature, then $\mathcal{K}$ is finitely axiomatizable.

K. Kearnes and R. McKenzie [13] developed a commutator theory for relative congruences in relatively congruence modular quasivarieties, with the expectation that it can be used to prove D. Pigozzi's conjecture; but the truth of this conjecture remains an open question.

We prove that D. Pigozzi's conjecture is true provided that $\mathcal{K}$ is additionally assumed to be included in a residually small congruence modular variety.

In view of R. McKenzie's negative solution to A. Tarski's problem (see R. McKenzie [20]) which asked if there is an algorithm to determine whether the variety generated by a finite set of finite algebras of finite signature is finitely axiomatizable, and the fact that the analogue of A. Tarski's problem for quasivarieties has not been solved yet, theorems like those of K. Baker, R. McKenzie, D. Pigozzi, and R. Willard have been gaining an extra meaning. All known theorems of this type provide sufficient conditions for a finitely generated variety or quasivariety of finite signature to be finitely axiomatizable. All the known sufficient conditions have been shown to be algorithmically verifiable. Continuing this tradition, we offer here, in Section 6, an algorithm for determining if a finite set of finite algebras generates a quasivariety satisfying $\operatorname{RSD}(\wedge)$.

2. More congruence properties, and relations amongst them. Besides the congruence properties introduced in the introduction, three properties of a more technical nature will also play a major part in our discussions. 
Let $\mathcal{K}$ be any quasivariety. We say that $\mathcal{K}$ satisfies the extension property, $\mathrm{EP}$, if and only if for all $\mathbf{A} \in \mathcal{K}$ the map $\theta \mapsto \bar{\theta}^{\mathcal{K}}$ is a lattice homomorphism of $\mathbf{C o n} \mathbf{A}$ onto $\operatorname{Con}_{\mathcal{K}} \mathbf{A}$. We say that $\mathcal{K}$ satisfies the weak extension property, WEP, if and only if for all $\mathbf{A} \in \mathcal{K}$ and $\alpha, \beta \in$ Con $\mathbf{A}$, if $\alpha \wedge \beta=0_{A}$ then $\bar{\alpha}^{\mathcal{K}} \wedge \bar{\beta}^{\mathcal{K}}=0_{A}$. We say that $\mathcal{K}$ satisfies the PCC, or has pseudo-complemented congruences, if for all $\mathbf{A} \in \mathcal{K}$ and $\alpha \in$ Con $\mathbf{A}$, there is a largest congruence $\beta \in$ Con $\mathbf{A}$ satisfying $\alpha \wedge \beta=0_{A}$ (i.e., $\beta$ is the pseudo-complement of $\alpha$ in Con A).

Together with the six properties defined in the introduction, we now have nine congruence properties to consider, as related below.

$\begin{aligned} \mathrm{CM} & \text { congruence modular } \\ \mathrm{CD} & \text { congruence distributive } \\ \mathrm{SD}(\wedge) & \text { congruence meet semi-distributive } \\ \mathrm{RCM} & \text { relatively congruence modular } \\ \mathrm{RCD} & \text { relatively congruence distributive } \\ \mathrm{RSD}(\wedge) & \text { relatively congruence meet semi-distributive } \\ \mathrm{EP} & \text { extension property } \\ \mathrm{WEP} & \text { weak extension property } \\ \mathrm{PCC} & \text { pseudo-complemented congruence lattices }\end{aligned}$

Whenever $\mathrm{P}$ is any one of these properties, we shall write $\mathcal{K} \models \mathrm{P}$ to denote that $\mathcal{K}$ satisfies $\mathrm{P}$.

Extending Willard's theorem to quasivarieties M. Maróti and R. McKenzie [17] proved that if $\mathcal{K}$ is a finitely generated quasivariety of finite signature which satisfies both PCC and WEP, then $\mathcal{K}$ is finitely axiomatizable. We shall prove that $\operatorname{RSD}(\wedge) \Leftrightarrow \mathrm{PCC}+$ WEP. Thus we shall obtain the full version of Willard's theorem for quasivarieties.

We also require two properties of a different character.

Let $\mathcal{K}$ be any quasivariety. A finite set of pairs of terms,

$$
W=\left\{\left(s_{i}(x, y, z), t_{i}(x, y, z)\right): 0 \leq i<n\right\},
$$

is said to be a set of Willard terms for $\mathcal{K}$ if and only if the equations $s_{i}(x, y, x) \approx t_{i}(x, y, x)(0 \leq i<n)$ are valid in $\mathcal{K}$ and $\mathcal{K} \models(\forall x, y)\left[x \neq y \rightarrow \bigvee_{i<n}\left(s_{i}(x, x, y)=t_{i}(x, x, y) \leftrightarrow s_{i}(x, y, y) \neq t_{i}(x, y, y)\right)\right]$

We write $\mathcal{K} \models \mathrm{W}$ to denote that $\mathcal{K}$ has a set of Willard terms.

A finite set of pairs of terms

$$
D=\left\{\left(p_{i}(x, y, z, u), q_{i}(x, y, z, u)\right): 0 \leq i<n\right\}
$$


is said to be a set of quasi-Day terms for $\mathcal{K}$ if and only if the equations $p_{i}(x, y, y, x) \approx q_{i}(x, y, y, x) \quad$ and $\quad p_{i}(x, x, y, y) \approx q_{i}(x, x, y, y), \quad 0 \leq i<n$, are valid in $\mathcal{K}$ and

$$
\mathcal{K} \models(\forall x, y, z)\left[x \neq z \rightarrow \bigvee_{i<n} p_{i}(x, y, y, z) \neq q_{i}(x, y, y, z)\right] .
$$

Most of the facts stated in the next theorem can be found either in K. Kearnes and R. McKenzie [13] or in M. Maróti and R. McKenzie [17]. We believe that Theorem 1(4) is new. The results in the corollary may be partly new.

TheOREm 1. For any quasivariety $\mathcal{K}$ the following hold.

(1) $\mathrm{CD} \Leftrightarrow \mathrm{CM}+\mathrm{SD}(\wedge)$,

(2) $\mathrm{RCD} \Leftrightarrow \mathrm{RCM}+\mathrm{RSD}(\wedge)$,

(3) $\mathrm{PCC} \Leftrightarrow \mathrm{W}$ and $\mathrm{SD}(\wedge) \Rightarrow \mathrm{PCC}$,

(4) $\operatorname{RSD}(\wedge) \Leftrightarrow \mathrm{PCC}+\mathrm{WEP} \Leftrightarrow \mathrm{W}+\mathrm{WEP}$,

(5) $\mathrm{RCM} \Rightarrow \mathrm{EP} \Rightarrow \mathrm{WEP}$,

(6) $\mathrm{RCM} \Leftrightarrow \mathcal{K}$ has quasi-Day terms and satisfies WEP.

Corollary 2. Let $\mathcal{K} \subseteq \mathcal{L}$ be quasivarieties.

(i) If $\mathcal{L}$ is $\mathrm{RCM}$ then $\mathcal{K}$ is $\mathrm{RCM}$ iff $\mathcal{K}$ satisfies WEP.

(ii) If $\mathcal{L}$ is $\operatorname{RSD}(\wedge)$ then $\mathcal{K}$ is $\operatorname{RSD}(\wedge)$ iff $\mathcal{K}$ satisfies WEP.

(iii) If $\mathcal{L}$ is $\mathrm{RCM}$ and $\mathcal{K}$ is $\operatorname{RSD}(\wedge)$ then $\mathcal{K}$ is $\mathrm{RCD}$.

(iv) If $\mathcal{L}$ is $\operatorname{RSD}(\wedge)$ and $\mathcal{K}$ is $\mathrm{RCM}$ then $\mathcal{K}$ is $\mathrm{RCD}$.

We remark that a quasivariety $\mathcal{K}$ is $\mathrm{CM}$ iff the variety $\mathcal{L}=\mathrm{H}(\mathcal{K})$ is $\mathrm{CM}$ and that here, $\mathcal{L} \models \mathrm{CM}$ iff $\mathcal{L} \models \mathrm{RCM}$. The analogous statements hold for the properties $\mathrm{CD}$ and $\operatorname{SD}(\wedge)$. Consequently, each of the four assertions of the corollary remains true when the hypothesis $\mathcal{L} \models \operatorname{RCM}$ or $\mathcal{L} \models \operatorname{RSD}(\wedge)$ is replaced by $\mathcal{K} \models \mathrm{CM}$ or, respectively, $\mathcal{K} \models \mathrm{SD}(\wedge)$.

Proof of Theorem 1. (1) and (2): It is a well-known fact that any lattice is distributive iff it is both modular and meet (or join) semi-distributive.

(3): The part PCC $\Leftrightarrow \mathrm{W}$ was proved in M. Maróti and R. McKenzie [17] (see Theorem 2.3 of [17]). The part $\mathrm{SD}(\wedge) \Rightarrow \mathrm{PCC}$ is an easy consequence of the fact that every algebraic meet semi-distributive lattice is pseudo-complemented.

(4): We prove $\operatorname{RSD}(\wedge) \Leftrightarrow \mathrm{PCC}+\mathrm{WEP}$ and obtain $\operatorname{RSD}(\wedge) \Leftrightarrow \mathrm{W}+\mathrm{WEP}$ as a consequence of $(3)$.

Suppose that $\mathcal{K} \models \mathrm{PCC}+\mathrm{WEP}$. Let $\mathbf{A} \in \mathcal{K}, \alpha, \beta, \gamma \in \mathbf{C o n}_{\mathcal{K}} \mathbf{A}$, and $\alpha \wedge \beta=\alpha \wedge \gamma=0_{A}$. By PCC, $\alpha \wedge(\beta \vee \gamma)=0_{A}$, which, as $\alpha \in \mathbf{C o n}_{\mathcal{K}} \mathbf{A}$ and WEP, gives $\alpha \wedge(\beta \vee \mathcal{K} \gamma)=0_{A}$. Thus $\mathcal{K} \models \operatorname{RSD}(\wedge)$.

Next, we suppose that $\mathcal{K}$ satisfies $\operatorname{RSD}(\wedge)$ and we show that $\mathcal{K}$ has Willard terms, which, by (3), will give $\mathcal{K} \models$ PCC. Let $\mathbf{F}=\mathbf{F}_{\mathcal{K}}(3)$ be the free 
algebra in $\mathcal{K}$ freely generated by $x, y, z$. Let $\alpha=\mathrm{Cg}_{\mathbf{F}}(x, z)$, the congruence generated by the pair $(x, z)$; and let $\beta=\mathrm{Cg}_{\mathbf{F}}(x, y)$ and $\gamma=\mathrm{Cg}_{\mathbf{F}}(y, z)$. Notice that each of $\alpha, \beta, \gamma$ is the kernel of an endomorphism of $\mathbf{F}$, and hence $\{\alpha, \beta, \gamma\} \subseteq \operatorname{Con}_{\mathcal{K}}$ F. Put

$$
\begin{aligned}
\beta_{0} & =\beta, \\
\gamma_{0} & =\gamma, \\
\beta_{n+1} & =\beta \vee^{\mathcal{K}}\left(\alpha \wedge \gamma_{n}\right), \\
\gamma_{n+1} & =\gamma \vee^{\mathcal{K}}\left(\alpha \wedge \beta_{n}\right) .
\end{aligned}
$$

This defines increasing sequences $\left\langle\beta_{n}: n \in \omega\right\rangle,\left\langle\gamma_{n}: n \in \omega\right\rangle$ in $\operatorname{Con}_{\mathcal{K}} \mathbf{F}$. Then $\beta_{\infty}=\bigvee_{n} \beta_{n}$ and $\gamma_{\infty}=\bigvee_{n} \gamma_{n}$ are $\mathcal{K}$-congruences. By join-continuity, $\alpha \wedge \beta_{\infty}=\alpha \wedge \gamma_{\infty}$. Now since $\mathcal{K} \models \operatorname{RSD}(\wedge)$, we have $\alpha \wedge \beta_{\infty}=\alpha \wedge\left(\beta_{\infty} \vee^{\mathcal{K}} \gamma_{\infty}\right)$. Thus

$$
\alpha \wedge(\beta \vee \gamma) \leq \alpha \wedge\left(\beta_{\infty} \vee \gamma_{\infty}\right) \leq \alpha \wedge \beta_{\infty}
$$

showing that $(x, z) \in \beta_{\infty}$.

Now write

$$
M=\left\{(s(x, y, z), t(x, y, z)) \in F^{2}: \mathcal{K} \models s(x, y, x) \approx t(x, y, x)\right\} .
$$

In fact, $M=\alpha$. Suppose that $\mathbf{A} \in \mathcal{K}, a, b \in A$, and

$$
\mathbf{A} \models s(a, a, b)=t(a, a, b) \leftrightarrow s(a, b, b)=t(a, b, b) \text { for all }(s, t) \in M .
$$

This means that if $f_{0}, f_{1}$ are the homomorphisms $\mathbf{F} \rightarrow \mathbf{A}$ with

$$
\left(f_{0}(x), f_{0}(y), f_{0}(z)\right)=(a, a, b), \quad\left(f_{1}(x), f_{1}(y), f_{1}(z)\right)=(a, b, b),
$$

and $\beta^{\prime}=\operatorname{ker}\left(f_{0}\right), \gamma^{\prime}=\operatorname{ker}\left(f_{1}\right)$, then $\alpha \wedge \beta^{\prime}=\alpha \wedge \gamma^{\prime}$. Notice that $\beta^{\prime}, \gamma^{\prime} \in$ $\operatorname{Con}_{\mathcal{K}} \mathbf{F}$ and $\beta \leq \beta^{\prime}, \gamma \leq \gamma^{\prime}$. An easy calculation then shows inductively that

$$
\beta_{n} \leq \beta^{\prime}, \quad \gamma_{n} \leq \gamma^{\prime} \quad \text { for all } n .
$$

We conclude that $(x, z) \in \beta^{\prime}$ (since $(x, z) \in \beta_{\infty}$ ). But this just means that $a=b$ (i.e., $f_{0}(x)=f_{0}(z)$ ).

We have shown that

$$
\mathcal{K} \models(\forall x, z)\left[x \neq z \rightarrow \bigvee_{(s, t) \in M}(s(x, x, z)=(x, x, z) \leftrightarrow s(x, z, z) \neq t(x, z, z))\right]
$$

With a logical compactness argument, we deduce that the displayed statement remains true when $M$ is replaced by some finite subset $W$ of $M$. Clearly, $W$ is a set of Willard terms for $\mathcal{K}$.

This concludes our proof that $\operatorname{RSD}(\wedge) \Rightarrow \mathrm{W}$.

Finally, we prove that $\operatorname{RSD}(\wedge) \Rightarrow$ WEP. It will be a proof by contradiction. Suppose that $\mathcal{K} \models \operatorname{RSD}(\wedge)$, and that $\mathbf{A} \in \mathcal{K},\{\theta, \psi\} \subseteq \operatorname{Con} \mathbf{A}$, $\theta \wedge \psi=0_{A}$, and $\bar{\theta}^{\mathcal{K}} \wedge \bar{\psi}^{\mathcal{K}}>0_{A}$. If $\theta \wedge \bar{\psi}^{\mathcal{K}}=0_{A}$, replace $\theta$ by $\bar{\psi}^{\mathcal{K}}$, and replace $\psi$ by $\theta$. Thus we can assume that $\theta \wedge \psi=0_{A}$ and $\theta \wedge \bar{\psi}^{\mathcal{K}}>0_{A}$. 
Now let

$$
B=\left\{(x, y) \in A^{2} \text { : there exist } c, d \in A \text { so that } x \theta c \psi y \text { and } x \psi d \theta y\right\},
$$

that is, $B=(\theta \circ \psi) \cap(\psi \circ \theta)$. This is of course a subuniverse of $\mathbf{A} \times \mathbf{A}$ that includes $\theta \cup \psi$, and we write $\mathbf{B}$ for the corresponding algebra. If $(x, y) \in B$ and $c, d$ are as above, the elements $c, d$ are unique. We have four surjective homomorphisms $\mathbf{B} \rightarrow \mathbf{A}$ :

$$
\begin{array}{rlrl}
\pi_{0}(x, y) & =x ; & \\
\pi_{1}(x, y) & =y ; & \\
\varphi(x, y) & =c & & \text { where } x \theta c \psi y \\
\varphi^{\prime}(x, y) & =d & & \text { where } x \psi d \theta y .
\end{array}
$$

The kernels of these homomorphisms are, of course, $\mathcal{K}$-congruences of $\mathbf{B}$. They are

$$
\begin{aligned}
0_{0} & =\operatorname{ker}\left(\pi_{0}\right) ; \\
0_{1} & =\operatorname{ker}\left(\pi_{1}\right) ; \\
\theta_{0} \wedge \psi_{1} & =\operatorname{ker}(\varphi) ; \\
\psi_{0} \wedge \theta_{1} & =\operatorname{ker}\left(\varphi^{\prime}\right) .
\end{aligned}
$$

Thus

$$
\begin{aligned}
& \alpha=\operatorname{ker}(\varphi) \wedge \operatorname{ker}\left(\pi_{1}\right)=\theta_{0} \wedge 0_{1}, \\
& \beta=\operatorname{ker}\left(\varphi^{\prime}\right)=\psi_{0} \wedge \theta_{1}, \\
& \gamma=\operatorname{ker}\left(\pi_{0}\right) \wedge \operatorname{ker}(\varphi)=0_{0} \wedge \psi_{1}
\end{aligned}
$$

are $\mathcal{K}$-congruences of $\mathbf{B}$.

Obviously ( since $\theta \wedge \psi=0_{A}$ ) we have $\alpha \wedge \beta=\alpha \wedge \gamma=0_{B}$. Since $\mathcal{K} \models \operatorname{RSD}(\wedge)$, it follows that $\alpha \wedge \overline{\beta \vee \gamma}^{\mathcal{K}}=0_{B}$. We can calculate $\beta \vee \gamma$ as

$$
\beta \vee \gamma=\psi_{0}
$$

To see this, note first that $\beta \cup \gamma \subseteq \psi_{0}$. Next, suppose that $\left(\left(x_{0}, y_{0}\right),\left(x_{1}, y_{1}\right)\right) \in$ $\psi_{0}$. This means simply that $\left(x_{0}, x_{1}\right) \in \psi$. We can calculate:

$$
\left(x_{0}, y_{0}\right) \gamma\left(x_{0}, \varphi\left(x_{0}, y_{0}\right)\right) \beta\left(x_{1}, x_{0}\right) \gamma\left(x_{1}, x_{1}\right) \beta\left(x_{1}, \varphi\left(x_{1}, y_{1}\right)\right) \gamma\left(x_{1}, y_{1}\right) .
$$

Next, note that from the isomorphism $\mathbf{B} / \psi_{0} \cong \mathbf{A} / \psi$, it follows that the interval $1_{A} / \psi$ in $\mathbf{C o n} \mathbf{A}$ is naturally isomorphic to the interval $1_{B} / \psi_{0}$ in Con $\mathbf{B}$ via the map $\tau \mapsto \tau_{0}$, where for all such $\tau, \mathbf{A} / \tau \cong \mathbf{B} / \tau_{0}$. From this, we deduce that $\left(\bar{\psi}^{\mathcal{K}}\right)_{0}=\bar{\psi}_{0}{ }^{\mathcal{K}}$. Now

$$
\alpha \wedge \bar{\psi}_{0}^{\mathcal{K}}=\alpha \wedge \overline{\beta \vee \gamma}^{\mathcal{K}}=0_{B}
$$


since $\mathcal{K} \models \operatorname{RSD}(\wedge)$. But on the other hand, we have some $(x, y) \in \theta \wedge \bar{\psi}^{\mathcal{K}}$, $x \neq y$. Then $(x, y),(y, y) \in B$ and obviously,

$$
((x, y),(y, y)) \in \alpha \wedge \bar{\psi}_{0}^{\mathcal{K}},
$$

which contradicts the displayed equations just above. This ends our proof that $\operatorname{RSD}(\wedge) \Rightarrow \mathrm{WEP}$.

Our proof of (4) is now complete. Statement (5) is Theorem 1.1 in K. Kearnes and R. McKenzie [13]. Theorem 4.1 in the same paper demonstrates that RCM is equivalent to the conjunction of EP and the existence of quasi-Day terms. But in the paragraph immediately following the conclusion of their proof of Theorem 4.1, those authors point out that our stronger Theorem 1(6) is true and show how to prove it.

Proof of Corollary 2. Assume that $\mathcal{K} \subseteq \mathcal{L}$ are quasivarieties. It is clear that if $\mathcal{L}$ satisfies PCC (equivalently, W), or has quasi-Day terms, then $\mathcal{K}$ has the same property. Thus (i) and (ii) follow immediately from Theorem 1(4), (6). Statements (iii) and (iv) then follow from the equivalence $\mathrm{RCD} \Leftrightarrow \mathrm{RCM}+\operatorname{RSD}(\wedge)$. Since we did not prove Theorem $1(6)$, and the proof given in K. Kearnes and R. McKenzie [13] is rather involved, we shall now give a direct proof of the fact that if $\mathcal{L}$ is relatively congruence modular and $\mathcal{K}$ has the weak extension property, then $\mathcal{K}$ is relatively congruence modular.

Assume that $\mathcal{L}$ has RCM and $\mathcal{K}$ has WEP. To see that $\mathcal{K}$ has RCM, let $\mathbf{A} \in \mathcal{K}$ and $\alpha, \beta, \gamma \in \operatorname{Con}_{\mathcal{K}} \mathbf{A}$ with $\alpha \geq \beta$. We must prove that $\alpha \wedge(\beta \vee \mathcal{K} \gamma)$ $=\beta \vee^{\mathcal{K}}(\alpha \wedge \gamma)$. By replacing $\beta$ with $\beta \vee^{\mathcal{K}}(\alpha \wedge \gamma)$, we can assume that $\alpha \wedge \gamma \leq \beta$. Next, replacing $\mathbf{A}$ by $\mathbf{A} /(\alpha \wedge \gamma)$ (an algebra in $\mathcal{K})$ and replacing $\alpha$ by $\alpha /(\alpha \wedge \gamma), \beta$ by $\beta /(\alpha \wedge \gamma), \gamma$ by $\gamma /(\alpha \wedge \gamma)$, we can assume that $\alpha \wedge \gamma=0_{A}$. Note that $\{\alpha, \beta, \gamma\} \subseteq \operatorname{Con}_{\mathcal{L}} \mathbf{A}$ and we do have $\alpha \wedge\left(\beta \vee^{\mathcal{L}} \gamma\right)=\beta \vee^{\mathcal{L}}(\alpha \wedge \gamma)=\beta$. Now $\alpha, \beta$ are in $\operatorname{Con}_{\mathcal{K}} \mathbf{A}$ and if $\tau=\beta \vee^{\mathcal{L}} \gamma$, then obviously $\bar{\tau}^{\mathcal{K}}=\beta \vee^{\mathcal{K}} \gamma$. Applying the WEP in the algebra $\mathbf{A} / \beta$, it follows from $\alpha \wedge \tau=\beta$ that $\alpha \wedge \bar{\tau}^{\mathcal{K}}=\beta$-i.e., $\alpha \wedge\left(\beta \vee^{\mathcal{K}} \gamma\right)=\beta=\beta \vee^{\mathcal{K}}(\alpha \wedge \gamma)$, as desired.

We recommend to the reader the proof, in K. Kearnes and R. McKenzie [13] (Theorem 1.1), that RCM $\Rightarrow$ WEP (and in fact implies EP). It is a nice argument.

3. Quasi-Maltsev type characterization of $\operatorname{RSD}(\wedge)$. As in K. Kearnes and R. McKenzie [13], by a quasi-Maltsev condition we mean a condition expressed as the disjunction of conditions each of which postulates the existence of a finite set of terms satisfying certain quasi-equations. In general, any quasi-Maltsev condition alone cannot characterize $\operatorname{RSD}(\wedge)$. The reason is that an $\operatorname{RSD}(\wedge)$ quasivariety may have a subquasivariety that is not $\operatorname{RSD}(\wedge)$. However, $\operatorname{RSD}(\wedge)$ can be characterized by the conjunction of a 
quasi-Maltsev condition and the weak extension property, as shown by Theorem 3 below. A similar characterization for RCM quasivarieties was given in K. Kearnes and R. McKenzie [13]; see Theorem 1(6) in the present paper.

Let $\mathcal{K}$ be a quasivariety and $\mathbf{F}=\mathbf{F}_{\mathcal{K}}(3)$ be the free algebra in $\mathcal{K}$ freely generated by $x, y, z$. Let $\alpha=\operatorname{Cg}_{\mathbf{F}}(x, z), \beta=\operatorname{Cg}_{\mathbf{F}}(x, y)$, and $\gamma=\operatorname{Cg}_{\mathbf{F}}(y, z)$. In the proof of Theorem 1, we defined the following two sequences of congruences.

$$
\begin{aligned}
\beta_{0} & =\beta, \\
\gamma_{0} & =\gamma, \\
\beta_{n} & =\beta \vee \mathcal{K}\left(\alpha \wedge \gamma_{n-1}\right), \\
\gamma_{n} & =\gamma \vee^{\mathcal{K}}\left(\alpha \wedge \beta_{n-1}\right) .
\end{aligned}
$$

We will refer to these congruences again; see Theorem 3 and its proof given below.

A tree is a finite ordered set $(T, \leq)$ having a smallest element, called the root of $T$, and the property that every set of the form $r \downarrow=\{x \in T: x \leq r\}$ is a chain in $T$. If $t<r$ and $r \downarrow=\{r\} \cup t \downarrow$, then $r$ is called a child of $t$. A leaf of $T$ is a maximal element in $(T, \leq)$. For $t \in T$, we set $h(t)=|t \downarrow|-1$ and $C_{T}(t)=\{r \in T: r$ is a child of $t\}$. We also set $h(T)=\max \{h(t): t \in T\}$. A tree is said to be non-trivial if $h(T)>0$.

TheOREM 3. For any quasivariety $\mathcal{K}$ the following conditions are equivalent:

(i) $\mathcal{K}$ is $\operatorname{RSD}(\wedge)$;

(ii) $\mathcal{K}$ satisfies $\mathrm{WEP}$ and $(x, z) \in \beta_{n}$ for some $n \in \omega \backslash\{0\}$;

(iii) $\mathcal{K}$ satisfies WEP and there exists a finite set $\Delta=\left\{\left(p_{t}, q_{t}\right): t \in T\right\}$ $\subseteq F^{2}$ where the index set $T$ of $\Delta$ has the structure of a non-trivial tree such that, for every $t \in T$, the following equations and quasiequations are universally valid in $\mathcal{K}$ :

(1) If $t$ is the root of $T$, then $p_{t}(x, y, z) \approx x$ and $q_{t}(x, y, z) \approx z$.

(2) If $t$ is not a leaf in $T$, then $p_{t}(x, y, x) \approx q_{t}(x, y, x)$ and $\bigwedge\left\{p_{r}(x, y, z) \approx q_{r}(x, y, z): r \in C_{T}(t)\right\} \rightarrow p_{t}(x, y, z) \approx q_{t}(x, y, z)$.

(3) If $0<h(t)<h(T)$ and $t$ is a leaf in $T$, then $p_{t}(x, x, y) \approx$ $q_{t}(x, x, y)$ when $h(t)$ is odd, and $p_{t}(x, y, y) \approx q_{t}(x, y, y)$ when $h(t)$ is even.

(4) If $h(t)=h(T)$, then either $p_{t}(x, x, y) \approx q_{t}(x, x, y)$, or $p_{t}(x, y, x)$ $\approx q_{t}(x, y, x)$ and $p_{t}(x, y, y) \approx q_{t}(x, y, y)$ when $h(T)$ is odd, and either $p_{t}(x, y, y) \approx q_{t}(x, y, y)$, or $p_{t}(x, y, x) \approx q_{t}(x, y, x)$ and $p_{t}(x, x, y) \approx q_{t}(x, x, y)$ when $h(T)$ is even.

Proof. (i) $\Rightarrow$ (ii): It follows from (i) that $\alpha \wedge(\beta \vee \gamma) \leq \beta_{\infty}$ - see the proof of Theorem 1. This gives $(x, z) \in \beta_{n}$ for some $n \in \omega \backslash\{0\}$. 
(ii) $\Rightarrow$ (iii): Assume that $\mathcal{K}$ satisfies WEP, $n>0$, and $(x, z) \in \beta_{n}$. The idea of the proof of this implication is simple. We begin with the following claim, which is a consequence of the definitions of $\beta_{k}$ and $\gamma_{k}$ and a compactness argument.

Claim 1. For $k>0$ and 3-ary terms $p$ and $q$, if $(p, q) \in \beta_{k}($ or $(p, q) \in$ $\gamma_{k}$, respectively) then there are finite sets $A \subseteq \beta$ and $B \subseteq \alpha \cap \gamma_{k-1}$ (or $A \subseteq \gamma$ and $B \subseteq \alpha \cap \beta_{k-1}$, respectively) such that $\mathcal{K} \models \bigwedge\{r \approx s:(r, s) \in A \cup B\} \rightarrow$ $p \approx q$.

Now for every odd integer $i$ with $0 \leq i<n$, choose (using the claim) a mapping $\sigma \mapsto\left(A_{i}(\sigma), B_{i}(\sigma)\right)$ defined on $\gamma_{n-i}$ so that for $\sigma=(p, q) \in$ $\gamma_{n-i}, A_{i}(\sigma)$ is a finite subset of $\gamma, B_{i}(\sigma)$ is a finite subset of $\alpha \cap \beta_{n-i-1}$, $A_{i}(\sigma) \cap \alpha \cap \beta_{n-i-1}=\emptyset$, and

$$
\mathcal{K} \models \bigwedge\left\{r \approx s:(r, s) \in A_{i}(\sigma) \cup B_{i}(\sigma)\right\} \rightarrow p \approx q .
$$

By placing $(x, x)$ in $B_{i}(\sigma) \backslash A_{i}(\sigma)$, we can assume that $B_{i}(\sigma) \neq \emptyset$. For every even integer $i$ with $0 \leq i<n$, choose a mapping $\sigma \mapsto\left(A_{i}(\sigma), B_{i}(\sigma)\right)$ defined on $\beta_{n-i}$ so that for $\sigma=(p, q) \in \beta_{n-i}, A_{i}(\sigma)$ is a finite subset of $\beta, B_{i}(\sigma)$ is a finite non-empty subset of $\alpha \cap \gamma_{n-i-1}, A_{i}(\sigma) \cap \alpha \cap \gamma_{n-i-1}=\emptyset$, and

$$
\mathcal{K}=\bigwedge\left\{r \approx s:(r, s) \in A_{i}(\sigma) \cup B_{i}(\sigma)\right\} \rightarrow p \approx q .
$$

The labeled tree $T$ we shall use to satisfy (iii) is now determined. We construct it as follows.

For the elements of $T$ we take all finite sequences $t=\left(\sigma_{0}, \ldots, \sigma_{i}\right)$ of elements of $F^{2}$ satisfying: $0 \leq i \leq n ; \sigma_{0}=(x, z)$; for $0 \leq j<i, \sigma_{j} \in \beta_{n-j}$ if $j$ is even, $\sigma_{j} \in \gamma_{n-j}$ if $j$ is odd, and $\sigma_{j} \in \alpha$ if $0<j<i$; for $0 \leq j<i$, $\sigma_{j+1} \in A_{j}\left(\sigma_{j}\right) \cup B_{j}\left(\sigma_{j}\right)$. (Note that these conditions imply that if $0 \leq j<i-1$ then $\sigma_{j+1} \in B_{j}\left(\sigma_{j}\right)$, but $\sigma_{i} \in A_{i-1}\left(\sigma_{i-1}\right)$ is allowed when $A_{i-1}\left(\sigma_{i-1}\right) \neq \emptyset$.)

$T$ is ordered by putting $t \leq t^{\prime}$ iff $t$ is an initial segment of $t^{\prime}$. This obviously makes $T$ into a rooted tree with root $\perp=((x, z))$. For $t=\left(\sigma_{0}, \ldots, \sigma_{i}\right) \in T$ we put $p_{t}(x, y, z)=p$ and $q_{t}(x, y, z)=q$ where $\sigma_{i}=(p, q)$. The tree $T$ has height $n$. We leave to the reader the straightforward proof that $T$ with the labels $\left(p_{t}, q_{t}\right), t \in T$, satisfies (iii).

(iii) $\Rightarrow$ (i): Assume (iii). In particular, assume that $\mathcal{K}$ satisfies WEP and that we have a non-trivial tree $T$ with labels $\left(p_{t}, q_{t}\right), t \in T$, that satisfies (1)-(4) of statement (iii). Since $\mathcal{K} \models \mathrm{WEP}$, in order to prove (i) it is enough, by Theorem $1(4)$, to show that $\mathcal{K}$ has Willard terms.

Claim 2. $W=\left\{\left(p_{t}, q_{t}\right): t \in T\right.$ and $\left.\mathcal{K} \models p_{t}(x, y, x) \approx q_{t}(x, y, x)\right\}$ is a set of Willard terms for $\mathcal{K}$. 
To prove this claim, let $\mathbf{A} \in \mathcal{K},\{a, b\} \subseteq A$, and suppose that for all $\left(p_{t}, q_{t}\right) \in W$ we have

$$
p_{t}(a, a, b)=q_{t}(a, a, b) \leftrightarrow p_{t}(a, b, b)=q_{t}(a, b, b) .
$$

We must show that $a=b$.

If $n=h(T)$, let $I$ be the set of all $i, 0 \leq i \leq n$, such that: (I) if $i$ is odd and $i=h(t)$, then $p_{t}(a, a, b)=q_{t}(a, a, b)$; and (II) if $i$ is even and $i=h(t)$, then $p_{t}(a, b, b)=p_{t}(a, b, b)$. We show that $I=\{0,1, \ldots, n\}$, by reverse induction on $i$, starting at $i=n$ and working down to $i=0$. As a result we will find that $0 \in I$, implying that, with $\perp$ being the root of $T$, $a=p_{\perp}(a, b, b)=q_{\perp}(a, b, b)=b$, as desired.

For the base step of the induction, consider a vertex $t$ of height $n$. If $n$ is odd, and $p_{t}(x, x, y) \approx q_{t}(x, x, y)$ is not a valid equation of $\mathcal{K}$, then by (4) of Theorem 3(iii), $\left(p_{t}, q_{t}\right) \in W$ and $p_{t}(a, b, b)=q_{t}(a, b, b)$. In this case, $p_{t}(a, a, b)=q_{t}(a, a, b)$ by our assumption about $a, b$. On the other hand, if $p_{t}(x, x, y) \approx q_{t}(x, x, y)$ is an equation of $\mathcal{K}$, then of course $p_{t}(a, a, b)=$ $q_{t}(a, a, b)$. This shows that $n \in I$ if $n$ is odd. The argument for the case of even $n$ is analogous, and also uses (4) of Theorem 3(iii).

Now assume that $0 \leq i<n$ and $i+1 \in I$. We show that $i \in I$. So let $t$ be a vertex of $T$ of height $i$. Let, say, $i$ be even. First, assume that $t$ is not a leaf. Now for every child $r$ of $t$ in $T, h(r)=i+1$ and so $p_{r}(a, a, b)=$ $q_{r}(a, a, b)$ by the inductive assumption. Then, by (2) of Theorem 3(iii), we have $p_{t}(a, a, b)=q_{t}(a, a, b)$, and also $\left(p_{t}, q_{t}\right) \in W$. Thus it follows that $p_{t}(a, b, b)=q_{t}(a, b, b)$, as required. The case where $i$ is odd and $t$ is not a leaf yields to a similar argument.

Finally, assume that $t$ is a leaf of height $i$. Then by (3) of Theorem 3(iii), we have $p_{t}(a, a, b)=q_{t}(a, a, b)$ if $i$ is odd, and if $i$ is even, then $p_{t}(a, b, b)=$ $q_{t}(a, b, b)$. This concludes our proof that $i+1 \in I$ implies $i \in I$; and that concludes our proof of Theorem 3.

COROllary 4. A quasivariety $\mathcal{K}$ has Willard terms if and only if it admits a labeled tree satisfying condition (iii) of Theorem 3 . If $T$, with labels $\left(p_{t}, q_{t}\right), t \in T$, is such a tree for $\mathcal{K}$, then $W=\left\{\left(p_{t}, q_{t}\right): t \in T\right.$ and $\mathcal{K} \models$ $\left.p_{t}(x, y, x) \approx q_{t}(x, y, x)\right\}$ is a set of Willard terms for $\mathcal{K}$.

Proof. Claim 2 in the proof of Theorem 3 shows that if $T$ is such a tree for $\mathcal{K}$ then $W$ is a set of Willard terms for $\mathcal{K}$. Conversely, it is proved in M. Maróti and R. McKenzie [17] that a quasivariety has Willard terms iff it has pseudo-complemented congruence lattices, and consequently the possession of Willard terms implies that $(x, z) \in \beta_{n}$ for some $n>0$.

The tree condition (iii) in Theorem 3 is similar to the condition shown by $\mathrm{R}$. Willard [26] to be equivalent to congruence meet semi-distributivity 
for a variety, and from which he extracted the existence of what we have called Willard terms.

4. Finite axiomatizability. Our purpose in this section is to prove a very general result about finite axiomatizability (Theorem 6 ), and use it to prove that every finitely generated quasivariety satisfying $\operatorname{RSD}(\wedge)$, and having finite signature, is finitely axiomatizable (Theorem 7). Using Theorem 4, we also partially prove D. Pigozzi's conjecture (Theorem 8).

Let us recall from the introduction some notable finite axiomatizability theorems. Suppose that $\mathcal{K}$ is a finitely generated quasivariety of finite signature. $K$. Baker [1], [2] proved that $\mathcal{K}$ is finitely axiomatizable provided $\mathcal{K}$ satisfies $\mathrm{CD}+\mathrm{H}(\mathcal{K})=\mathcal{K}$ (i.e., $\mathcal{K}$ is a congruence distributive variety). D. Pigozzi [25] proved more generally that $\mathcal{K}$ is finitely axiomatizable if $\mathcal{K}$ satisfies RCD. R. Willard [26] extended K. Baker's theorem in another direction: $\mathcal{K}$ is finitely axiomatizable if $\mathcal{K}$ satisfies $\mathrm{SD}(\wedge)+\mathrm{H}(\mathcal{K})=\mathcal{K}$. M. Maróti and R. McKenzie [17] obtained a somewhat complicated result (see their Theorem 7.1) that extends both theorems of D. Pigozzi and R. Willard. Our Theorem 7 below extends the theorems of D. Pigozzi and R. Willard, and is a cleaner, more refined version of the result of M. Maróti and R. McKenzie.

Let $\mathcal{K}$ and $\mathcal{L}$ be locally finite quasivarieties of finite signature such that $\mathcal{K}$ is properly included in $\mathcal{L}$. A finite algebra $\mathbf{A}$ is said to be $(\mathcal{K}, \mathcal{L})$-minimal if $\mathbf{A} \notin \mathcal{K}, \mathbf{A} \in \mathcal{L}$, and every proper subalgebra of $\mathbf{A}$ belongs to $\mathcal{K}$. For a $(\mathcal{K}, \mathcal{L})$-minimal algebra $\mathbf{A}$, we let $\Theta_{\mathcal{K}}^{\mathbf{A}}$ denote the smallest congruence on $\mathbf{A}$ such that $\mathbf{A} / \Theta_{\mathcal{K}}^{\mathbf{A}} \in \mathcal{K}$.

Obviously, the relation of isomorphism partitions the family of all $(\mathcal{K}, \mathcal{L})$ minimal algebras. Let $\mathcal{S}$ be a selector set of that partition.

For $\mathbf{A} \in \mathcal{S}$, let $a \mapsto x_{a}$ be a fixed one-to-one assignment of individual variables to the elements of $\mathbf{A}$. Let $D(\mathbf{A})$ denote the collection of all equations of the form $f\left(x_{a_{1}}, \ldots, x_{a_{n}}\right)=x_{f\left(a_{1}, \ldots, a_{n}\right)}$, where $f$ is an $n$-ary operation symbol of the signature of $\mathbf{A}$, and $a_{1}, \ldots, a_{n}$ are elements of $\mathbf{A}$. As the signature is finite and $\mathbf{A}$ is finite, $D(\mathbf{A})$ is finite. We set

$$
\Sigma_{\mathbf{A}}=\left\{\bigwedge D(\mathbf{A}) \rightarrow x_{a} \approx x_{b}: a \neq b \text { and }(a, b) \in \Theta_{\mathcal{K}}^{A}\right\} .
$$

For a set $\Gamma$ of quasi-identities, we let $\operatorname{Mod}_{\mathcal{L}} \Gamma$ denote the set of algebras of $\mathcal{L}$ in which the quasi-identities of $\Gamma$ are universally valid.

Proposition 5. $\mathcal{K}=\operatorname{Mod}_{\mathcal{L}}\left(\bigcup\left\{\Sigma_{\mathbf{A}}: \mathbf{A} \in \mathcal{S}\right\}\right)$.

Proof. $\subseteq$ : Let $\mathbf{B} \in \mathcal{K}$ and $\mathbf{A} \in \mathcal{S}$. Let $(a, b) \in \Theta_{\mathcal{K}}^{\mathbf{A}}$ be such that $a \neq b$. Let $x_{a} \mapsto v\left(x_{a}\right)$ be an assignment of values in $\mathbf{B}$ of $\left\{x_{a}: a \in A\right\}$ under which the equations of $D(\mathbf{A})$ are satisfied. Notice that the map $a \mapsto v\left(x_{a}\right)$, denoted by $\varphi$, is a homomorphism from $\mathbf{A}$ to $\mathbf{B}$. As $\mathbf{B} \in \mathcal{K}, \mathbf{A} / \operatorname{ker}(\varphi) \in \mathcal{K}$. 
So $\Theta_{\mathcal{K}}^{\mathbf{A}} \leq \operatorname{ker}(\varphi)$. This gives $\varphi(a)=\varphi(b)$. Thus $v\left(x_{a}\right)=v\left(x_{b}\right)$, proving that $\mathbf{B}=\bigwedge D(A) \rightarrow x_{a}=x_{b}$, i.e. $\mathbf{B} \in \operatorname{Mod}_{\mathcal{L}}\left(\bigcup\left\{\Sigma_{\mathbf{A}}: \mathbf{A} \in \mathcal{S}\right\}\right)$.

$\supseteq$ : Let $\mathbf{B} \in \mathcal{L}$ be finite and $\mathbf{B} \notin \mathcal{K}$. Then there is $\mathbf{A} \in \mathcal{S}$ that is isomorphic to a subalgebra of $\mathbf{B}$. As $\mathbf{A} \not \models \Sigma_{\mathbf{A}}, \mathbf{B} \not \models \Sigma_{\mathbf{A}}$. Thus $\mathbf{B} \notin$ $\operatorname{Mod}_{\mathcal{L}}\left(\bigcup\left\{\Sigma_{\mathbf{A}}: \mathbf{A} \in \mathcal{S}\right\}\right)$

TheOREm 6. Suppose that $\mathcal{K} \subseteq \mathcal{L} \subseteq \mathcal{L}^{\prime}$ are quasivarieties of finite signature such that $\mathcal{K}$ satisfies WEP and $\mathcal{L}^{\prime}$ is finitely generated. Then $\mathcal{K}$ is finitely axiomatizable relative to $\mathcal{L}$.

Proof. Let $n$ be an integer not less than the cardinality of any of the generators of $\mathcal{L}^{\prime}$. Let $m$ be the maximum size of an $n$-generated subalgebra of any algebra in $\mathcal{L}^{\prime}$. We may assume that $\mathcal{K} \neq \mathcal{L}$. We claim that any finite algebra in $\mathcal{L} \backslash \mathcal{K}$ has a subalgebra of at most $m$ elements that lies outside of $\mathcal{K}$. This will mean that the selector $\mathcal{S}$ as defined above is finite. This, in turn, by Proposition 5, will complete the proof.

To see the claim, we assume that $\mathbf{A}$ is a finite algebra in $\mathcal{L} \backslash \mathcal{K}$. For $X, Y \subseteq$ Con $\mathbf{A}$, we define $X \preceq Y$ iff for every $\alpha \in Y$ there exists $\beta \in X$ such that $\beta \leq \alpha$. Notice that $\preceq$ restricted to antichains in Con $\mathbf{A}$ is a partial order.

Let $X$ be an antichain in Con $\mathbf{A}$ whose intersection is $0_{A}$ and such that for each $\alpha \in X$, the quotient algebra $\mathbf{A} / \alpha$ has at most $n$ elements. Notice that such an $X$ exists for $\mathbf{A} \in \mathcal{L}$ since $n$ bounds the cardinality of the generators of $\mathcal{L}^{\prime}$. Now, among all antichains $Y$ in Con $\mathbf{A}$ with $X \preceq Y$ and with the intersection of $Y$ equal to $0_{A}$, we choose one, say $Z$, that is $\preceq$-maximal. It follows from the choice of $Z$ that each member of $Z$ is a meet-irreducible element of Con $\mathbf{A}$. Since $\mathbf{A}$ is outside of $\mathcal{K}$, we can choose $\gamma$ in $Z$ so that $\mathbf{A} / \gamma \notin \mathcal{K}$. Let $\delta$ be the unique cover of $\gamma$ in Con $\mathbf{A}$. Since, by the choice, $Z$ is $\preceq$-maximal, there is a pair $(a, b)$ in $A \times A$ such that $(a, b) \in \delta \wedge \bigwedge(Z \backslash\{\gamma\})$ and $a \neq b$. Choose $a_{0}, \ldots, a_{k-1}$ in $A$ to be a selector set for all the $\gamma$-equivalence classes in $A$ with $a_{0}=a$ and $a_{1}=b$. (Note that $2 \leq k$ since $(a, b) \notin \gamma$.) As $X \preceq Z$, it follows that $k \leq n$.

Let $\mathbf{B}$ be the subalgebra of $\mathbf{A}$ generated by $a_{0}, \ldots, a_{k-1}$. As $k \leq n$, $\mathbf{B}$ has at most $m$ elements. Let $\gamma^{\prime}$ and $\sigma$ denote the restrictions of $\gamma$ and $\bigwedge(Z \backslash\{\gamma\})$ to the algebra $\mathbf{B}$, respectively. Obviously, $\gamma^{\prime} \wedge \sigma=0_{B}$. We now want to show that $\mathbf{B} \notin \mathcal{K}$. To this end, suppose otherwise that $\mathbf{B} \in \mathcal{K}$. Then, by WEP of $\mathcal{K}$, there are $\mathcal{K}$-congruences $\gamma_{\mathcal{K}}^{\prime}$ and $\sigma_{\mathcal{K}}$ on $\mathbf{B}$ that extend $\gamma^{\prime}$ and $\sigma$, respectively, and are such that $\gamma_{\mathcal{K}}^{\prime} \wedge \sigma_{\mathcal{K}}=0_{B}$. As $a, b \in B$ and $(a, b) \in \bigwedge(Z \backslash\{\gamma\})$, it follows that $(a, b) \in \sigma \leq \sigma_{\mathcal{K}}$. This implies $(a, b) \notin \gamma_{\mathcal{K}}^{\prime}$.

Since $\mathbf{B}$ is a subalgebra of $\mathbf{A}$ generated by a selector set for all the $\gamma$ equivalence classes in $\mathbf{A}$, it follows that $\mathbf{B} / \gamma^{\prime}$ is isomorphic to $\mathbf{A} / \gamma$. Thus $\gamma^{\prime}$ has the unique cover in Con $\mathbf{B}$ to which $(a, b)$ belongs; recall that $a_{0}=a$ and $a_{1}=b$. This implies that $\gamma_{\mathcal{K}}^{\prime}=\gamma^{\prime}$ for otherwise $\gamma_{\mathcal{K}}^{\prime}$ would be above this 
unique cover and as a consequence $(a, b)$ would belong to $\gamma_{\mathcal{K}}^{\prime}$. But $\mathbf{A} / \gamma \notin \mathcal{K}$ and so $\mathbf{B} / \gamma^{\prime} \notin \mathcal{K}$. Hence $\mathbf{B} / \gamma_{\mathcal{K}}^{\prime} \notin \mathcal{K}$, a contradiction. Thus $\mathbf{B} \notin \mathcal{K}$.

We remark that in place of assuming $\mathcal{K}$ satisfies WEP in Theorem 6 it would suffice to assume, if $\mathcal{L}^{\prime}$ is generated by algebras of at most $n$ elements, that for every $n$-generated algebra $\mathbf{A} \in \mathcal{K}$ and for all $\alpha, \beta \in \operatorname{Con} \mathbf{A}$, if $\alpha \wedge \beta=0_{A}$ then $\bar{\alpha}^{\mathcal{K}} \wedge \bar{\beta}^{\mathcal{K}}=0_{A}$. (An examination of the proof reveals that this is all we used.)

In view of the fact that $\operatorname{RSD}(\wedge) \Leftrightarrow \mathrm{PCC}+\mathrm{WEP}$ for quasivarieties (Theorem 1(4)), our next theorem is clearly identical with Corollary 6.4 in M. Maróti and R. McKenzie [17]. Those authors, however, did not know this characterization of $\operatorname{RSD}(\wedge)$, and we wish to present a somewhat cleaner proof of the result than they gave. Nevertheless, we shall have to use without proof one of their results.

THEOREM 7. Every finitely generated quasivariety of finite signature satisfying $\operatorname{RSD}(\wedge)$ is finitely axiomatizable.

Proof. Let $\mathcal{K}$ be a finitely generated quasivariety of finite signature, satisfying $\operatorname{RSD}(\wedge)$. By Theorem 1(4) above, $\mathcal{K}$ satisfies PCC and WEP. Now, Corollary 5.4 in M. Maróti and R. McKenzie [17] and its proof establish that $\mathcal{K} \subseteq \mathcal{L} \subseteq \mathcal{L}^{\prime}$ for a certain finitely axiomatizable quasivariety $\mathcal{L}$ and finitely generated quasivariety $\mathcal{L}^{\prime}$. Then it follows from Theorem 6 that $\mathcal{K}$ is finitely axiomatizable.

As $\operatorname{RSD}(\wedge) \Leftrightarrow \mathrm{PCC}+\mathrm{WEP}$, one may ask whether Theorem 7 remains true if $\operatorname{RSD}(\wedge)$ is replaced in it by PCC or by WEP. The answer is negative in the first case (see W. Dziobiak [8], J. Ježek, M. Maróti and R. McKenzie [12], and R. McKenzie [21]) but it is unknown to us in the second case.

THEOREM 8. If $\mathcal{K}$ is a finitely generated quasivariety of finite signature which is $\mathrm{RCM}$ and $\mathrm{H}(\mathcal{K})$ is $\mathrm{CM}$ and residually small, then $\mathcal{K}$ is finitely axiomatizable.

Proof. By Theorem 1(6), $\mathcal{K}=$ WEP. The result now follows from Theorem 6, R. McKenzie's theorem (see introduction), and a result of R. Freese and R. McKenzie [9] which says that every finitely generated and residually small $\mathrm{CM}$ variety of finite signature is of the form $\mathcal{L}=\operatorname{SP}(\mathcal{M})$ for some finite set $\mathcal{M}$ of finite algebras.

COROLlary 9. Let $\mathcal{M}$ be a finite set of finite algebras of finite signature from a $\mathrm{CM}$ variety. If $\mathrm{SP}(\mathrm{HS}(\mathcal{M}))$ is $\mathrm{RCM}$, then $\mathrm{SP}(\mathrm{HS}(\mathcal{M})$ ) is finitely axiomatizable.

Proof. Let $\mathbf{B} \in \mathrm{HS}(\mathcal{M})$ be subdirectly irreducible with monolith $\theta$. Let $\psi$ be a congruence on $\mathbf{B}$ such that the commutator $[\theta, \psi]$ calculated in the variety generated by $\mathcal{M}$ is equal to $0_{B}$. As $\mathrm{SP}(\mathrm{HS}(\mathcal{M}))$ is $\mathrm{RCM}$ and 
$0_{B}=[\theta, \psi]=[\theta \wedge \psi, \psi]$, it follows from the note following Theorem 3.1 of K. Kearnes and R. McKenzie [13] that $[\psi, \psi]=0_{B}$. Thus any subdirectly irreducible algebra $\mathbf{B}$ from $\mathrm{HS}(\mathcal{M})$ satisfies, for $\theta$ the monolith of $\mathbf{B}$ : If $\psi \in$ Con $\mathbf{B}$ and $[\theta, \psi]=0_{B}$, then $[\psi, \psi]=0_{B}$. This, by a result of $\mathrm{R}$. Freese and $\mathrm{R}$. McKenzie [9], gives that $\operatorname{HSP}(\mathcal{M})$ is residually small. Thus, by Theorem $8, \mathrm{SP}(\mathrm{HS}(\mathcal{M}))$ is finitely axiomatizable.

In the context of Theorem 8 , it is worthwhile to mention a result of D. Hobby and R. McKenzie [11, Chapter 10] which says that if $\mathcal{V}$ is a locally finite variety which is residually finite and satisfies some non-trivial congruence identity, then $\mathcal{V}$ is congruence modular.

5. Examples. This section contains four examples each of which provides a negative answer to some questions emerging naturally in the context of our work.

EXAMPLE 10. This is an example of a finitely generated quasivariety $\mathcal{K}$ that satisfies $\operatorname{RSD}(\wedge)+\neg \mathrm{EP}$. We define a five-element algebra $\mathbf{A}$ and a two-element algebra $\mathbf{B}=\mathbf{A} / \gamma$ for a certain congruence $\gamma$ of $\mathbf{A}$ and take $\mathcal{K}=\mathrm{SP}(\{\mathbf{A}, \mathbf{B}\})$. We put

$$
\mathbf{A}=\left\langle\{0, a, b, c, 1\}, \wedge, \overline{0}, \bar{a}, \bar{b}, \bar{c}, \overline{1}, f_{0}, f_{1}\right\rangle
$$

so that $\langle\{0, a, b, c, 1\}, \wedge\rangle$ is a semilattice with least and largest elements 0,1 and with $a \wedge b=c$, the five elements of $\mathbf{A}$ are denoted by constants in the signature of $\mathbf{A}$, and $f_{0}, f_{1}$ are unary operations defined by $f_{0}(b)=f_{0}(1)=c$ and $f_{0}(x)=0$ for $x \in\{0, a, c\}, f_{1}(a)=f_{1}(1)=c$ and $f_{1}(x)=0$ for $x \in\{0, b, c\}$. This algebra is subdirectly irreducible and its congruence lattice is an eight-element lattice. Its least non-zero congruence is the equivalence relation $\mu$ that has $\{0, c\}$ as its only non-singleton equivalence class. Another congruence is $\gamma$ that has $\{0, a, b, c\}$ and $\{1\}$ as equivalence classes. We take $\mathbf{B}=\mathbf{A} / \gamma$, and $\mathcal{K}=\operatorname{SP}(\{\mathbf{A}, \mathbf{B}\})$.

For any two distinct elements $e, e^{\prime}$ of $A$, and any $f, f^{\prime} \in A \backslash\{1\}$, both $\mathbf{A}$ and $\mathbf{B}$ satisfy the quasi-equation $\bar{e}=\bar{e}^{\prime} \rightarrow \bar{f}=\bar{f}^{\prime}$. Hence the lattice of $\mathcal{K}$-congruences of $\mathbf{A}$ consists of $0_{A}, \gamma$, and $1_{A}$. Among the other congruences of $\mathbf{A}$ are $\beta_{0}$ with equivalence classes $\{0, c, a\},\{b, 1\}$ and $\beta_{1}$ with equivalence classes $\{0, c, b\},\{a, 1\}$. We have $\beta_{0} \wedge \beta_{1}=\mu$ while $\bar{\beta}_{0}^{\mathcal{K}}=1_{A}=\bar{\beta}_{1}^{\mathcal{K}}$ and $\bar{\mu}^{\mathcal{K}}=\gamma$. Thus $\mathcal{K}$ does not have the EP.

Since members of $\mathcal{K}$ are expansions of semilattices, $\mathcal{K}$ does have the PCC, even the $\operatorname{SD}(\wedge)$. To be able to conclude that $\mathcal{K} \models \operatorname{RSD}(\wedge)$, we must show that $\mathcal{K} \models$ WEP.

To see this, let $\mathbf{C}$ be any finite member of $\mathcal{K}$. We may assume that $\mathbf{C}$ is a subalgebra of $\mathbf{A}^{I_{0}} \times \mathbf{B}^{I_{1}}$ for finite, pairwise disjoint sets $I_{0}, I_{1}$ and not isomorphic to a subalgebra of $\mathbf{A}^{I_{0}^{\prime}} \times \mathbf{B}^{I_{1}^{\prime}}$ for any $I_{0}^{\prime} \subseteq I_{0}, I_{1}^{\prime} \subseteq I_{1}$ with $I_{0}^{\prime} \neq I_{0}$ or $I_{1}^{\prime} \neq I_{1}$. 
It will suffice to prove the following: Let $0_{C} \prec \alpha$ be any atom in the congruence lattice of $\mathbf{C}$ and let $\beta$ be the pseudo-complement of $\alpha$-the largest congruence disjoint from $\alpha$; then $\beta$ is a $\mathcal{K}$-congruence of $\mathbf{C}$. For that purpose, we may choose $p, q \in C$ so that $(p, q) \in \alpha, q<p$ (in the semilattice order), and for all $p^{\prime}, q^{\prime} \in C$ with $q^{\prime} \leq p^{\prime}<p$ and $\left(p^{\prime}, q^{\prime}\right) \in \alpha$ we have $p^{\prime}=q^{\prime}$.

CASE 0: There is $i_{0} \in I_{0}$ with $p\left(i_{0}\right) \neq q\left(i_{0}\right)$. If $p\left(i_{0}\right) \in\{a, b, 1\}$, then one of the pairs $\left(f_{0}(p), f_{0}(q)\right)=\left(p^{\prime}, q^{\prime}\right)$ or $\left(f_{1}(p), f_{1}(q)\right)=\left(p^{\prime}, q^{\prime}\right)$ has $p^{\prime}>q^{\prime}$. Since in both cases, $p^{\prime}<p$, this is impossible. Thus $p\left(i_{0}\right) \in\{0, c\}$, forcing $\left(p\left(i_{0}\right), q\left(i_{0}\right)\right)=(c, 0)$. Now $(p \wedge \bar{c}, q \wedge \bar{c}) \in \alpha$, and it follows that $q<p \leq \bar{c}$.

Now let $\eta_{i_{0}}$ denote the kernel of the projection of $\mathbf{C}$ onto $\mathbf{A}$ at the $i_{0}$ th coordinate (the projection is onto since we have the constants in the signature), and let $\eta_{i_{0}}^{\prime}$ denote the kernel of the projection of $\mathbf{C}$ into $\mathbf{A}^{I_{0} \backslash\left\{i_{0}\right\}} \times \mathbf{B}^{I_{1}}$. By the minimality of our subdirect representation of $\mathbf{C}$, there must be a pair $(r, s) \in \eta_{i_{0}}^{\prime}, r \neq s$. We can assume that $s<r$. As above, we can also assume that $r \leq \bar{c}^{\mathbf{C}}$. Thus $\left(r\left(i_{0}\right), s\left(i_{0}\right)\right)=(c, 0)$. Now it is easy to verify that $p \geq r \wedge p>s \wedge p \geq r \wedge q$. Thus $(r \wedge p, s \wedge p) \in \alpha$. (By minimality of $p$, then $p=r \wedge p$.) The pair $(r \wedge p, s \wedge p)$ of functions, which differ only at $i_{0}$, must generate $\alpha$; hence $\alpha \leq \eta_{i_{0}}^{\prime}$. This implies that $\alpha \wedge \eta_{i_{0}}=0_{C}$, and so $\beta \geq \eta_{i_{0}}$. Now $\mathbf{C} / \eta_{i_{0}} \cong \mathbf{A}$ and so $\eta_{i_{0}}$ has a unique cover $\mu_{i_{0}} \in \mathbf{C o n} \mathbf{C}$, i.e. $(x, y) \in \mu_{i_{0}}$ iff $(x, y) \in C^{2}$ and $\left(x\left(i_{0}\right), y\left(i_{0}\right)\right) \in \mu$. Clearly, $(r \wedge p, s \wedge p) \in \mu_{i_{0}}$, hence $\beta \nsupseteq \mu_{i_{0}}$. So we conclude that $\beta=\eta_{i_{0}}$. But this implies that $\beta$ is a $\mathcal{K}$-congruence of $\mathbf{C}$, as we wanted to prove.

Finally, we consider

CASE 1: If $\eta_{I_{0}}$ is the kernel of the projection of $\mathbf{C}$ into $\mathbf{A}^{I_{0}}$ and $\eta_{I_{1}}$ is the kernel of the projection of $\mathbf{C}$ into $\mathbf{B}^{I_{1}}$, we have $\alpha \leq \eta_{I_{0}}$. In this case, $\alpha \wedge \eta_{I_{1}}=0_{C}$, implying that $\beta \geq \eta_{I_{1}}$. But since $\mathbf{C} / \eta_{I_{1}}$ is embeddable into $\mathbf{B}^{I_{1}}$, it is easy to see that every congruence of $\mathbf{C}$ containing $\eta_{I_{1}}$ is a $\mathcal{K}$-congruence. Thus $\beta$ is a $\mathcal{K}$-congruence, also in Case 1.

EXAMPLE 11. This is an example of a finitely generated quasivariety that satisfies $\operatorname{SD}(\wedge)$ and not WEP, and hence PCC and not $\operatorname{RSD}(\wedge)$. We remark that the examples of finitely generated quasivarieties of lattices that are not finitely axiomatizable supplied by, for example, V. P. Belkin [3] satisfy PCC and, as a consequence of our Theorems 1 and 7, cannot satisfy WEP. The example we now present is, however, much simpler to demonstrate.

We use a four-element algebra $\mathbf{A}=\langle\{0, a, b, 1\}, \wedge, \overline{0}, \bar{a}, \bar{b}, \overline{1}\rangle$. Here, $\langle A, \wedge\rangle$ is the four element meet-semilattice with two atoms $a, b$ and the other operations are the constants. This algebra has the congruence $\gamma$ with classes $\{0, a, b\},\{1\}$ and we put $\mathbf{B}=\mathbf{A} / \gamma$ and $\mathcal{K}=\mathrm{SP}(\{\mathbf{A}, \mathbf{B}\})$. A also has congruences $\beta_{0}$ with classes $\{0, a\},\{1, b\}$ and $\beta_{1}$ with classes $\{0, b\},\{1, a\}$. By an obvious argument very similar to the one we used in the last example, one 
shows that $\overline{\beta_{0}}{ }^{\mathcal{K}}=1_{A}={\overline{\beta_{1}}}^{\mathcal{K}}$. However, $\beta_{0} \wedge \beta_{1}=0_{A}$, and this shows that $\mathcal{K}$ fails to have WEP. This quasivariety does have $\operatorname{SD}(\wedge)$, due to the presence of the semilattice operation.

EXAMPLE 12. This is an example of a finitely generated quasivariety that satisfies $\mathrm{RCD}+\mathrm{CM}+\neg \mathrm{CD}$. We put $\mathcal{K}=\mathrm{SP}(\mathbf{A})$ where $\mathbf{A}$ is a six-element algebra which we now define:

$$
\mathbf{A}=\left\langle\{0,1,2, a, b, c\}, m, \wedge, \overline{0}, \overline{1}, \overline{2}, \bar{a}, \bar{b}, \bar{c}, F_{0}, F_{a}, F_{b}, F_{c}\right\rangle
$$

has six constants, a ternary operation $m$, binary operation $\wedge$, and four unary operations $F_{r}$ where $r \in U=\{0, a, b, c\}$. We define $F_{r}$ so that $F_{r}(r)=2$ and $F_{r}(x)=1$ for all $x \in A \backslash\{r\}$. Let $D=\{1,2\}$ and let $\langle U,+\rangle$ and $\langle D,+\rangle$ be Boolean groups with identity elements 0,1 respectively. We define $m(x, y, z)$ so that $m\left(x_{0}, x_{1}, x_{2}\right)=x_{0}+x_{1}+x_{2}$ if $\left\{x_{0}, x_{1}, x_{2}\right\}$ is a subset of $U$ or of $D$; and otherwise, if $\left|\left\{i: x_{i} \in U\right\}\right|=1$ and $\{p\}=\left\{x_{0}, x_{1}, x_{2}\right\} \cap U$, or if $\left|\left\{i: x_{i} \in D\right\}\right|=1$ and $\{p\}=\left\{x_{0}, x_{1}, x_{2}\right\} \cap D$, then we put $m\left(x_{0}, x_{1}, x_{2}\right)=p$. Thus $m$ is fully defined as an operation on $A$. We define $x \wedge y$ to be the minimum element in $\{x, y\} \cap D$ if $\{x, y\} \cap D$ is non-empty, and otherwise $x \wedge y=2$.

Now $m$ is a Maltsev operation, i.e., it satisfies the equations $m(x, y, y)=$ $x=m(y, y, x)$ on $A$. Thus $\mathcal{K}$ satisfies CM. The equivalence relation $\mu$ with one non-trivial block $\{1,2\}$ is a congruence of $\mathbf{A}$, in fact, the smallest nonzero congruence of $\mathbf{A}$. Besides $0_{A}, 1_{A}$ and $\mu$, this algebra has precisely four further congruences; they are the equivalence relations $\mu \cup \beta$ where $\beta$ is any non-zero congruence on $\langle U,+\rangle$. The congruence lattice of $\langle U,+\rangle$ is in this way embedded as an interval in Con $\mathbf{A}$. This shows that $\mathcal{K}$ does not satisfy $\mathrm{CD}$.

Finally, we must demonstrate that $\mathcal{K}=\mathrm{RCD}$. It will suffice to show that every finite algebra in $\mathcal{K}$ has a distributive lattice of $\mathcal{K}$-relative congruences. So let $\mathbf{B}$ be a finite algebra in $\mathcal{K}$. We can assume that $\mathbf{B} \subseteq \mathbf{A}^{n}$ where $n$ is a positive integer, and that for all $1 \leq m<n$, $\mathbf{B}$ is not embeddable into $\mathbf{A}^{m}$. For $0 \leq i<n$ let $0_{i}$ be the kernel of the projection homomorphism mapping of $\mathbf{B}$ to $\mathbf{A}$ at the $i$ th coordinate. Because $n$ has been chosen to be minimal, the set $P=\left\{0_{0}, \ldots, 0_{n-1}\right\}$ is an $n$-element subset of $\operatorname{Con}_{\mathcal{K}} \mathbf{B}$, and moreover, the set $C$ of all intersections of subsets of $P$ is a $2^{n}$-element subset of Con $\operatorname{Co}_{\mathcal{K}} \mathbf{B}$. Actually, if $\{\phi\} \cup \Gamma \subseteq P$ then $\phi \geq \bigwedge \Gamma$ iff $\phi \in \Gamma$. Our objective is to prove that $C=\operatorname{Con}_{\mathcal{K}} \mathbf{B}$. If this is true, then it easily follows that the map $\Gamma \mapsto \bigwedge \Gamma$ is an isomorphism of the Boolean lattice of all subsets of $P$ onto the lattice $\operatorname{Con}_{\mathcal{K}} \mathbf{B}$. In this way, $\operatorname{Con}_{\mathcal{K}} \mathbf{B}$ will be shown to be distributive.

To accomplish this objective, suppose that $\theta$ is an arbitrary member of $\operatorname{Con}_{\mathcal{K}} \mathbf{B}$. Set $T$ equal to the set of all $\eta \in P$ such that $\theta \leq \eta$, and put $\theta^{\prime}=\bigwedge T$. So $\theta \leq \theta^{\prime}$ and we have to prove that $\theta=\theta^{\prime}$. 
Claim 1. $D^{n} \subseteq B$.

To prove this, choose any $0 \leq i<n$. Now since $0_{i} \nsupseteq \bigwedge\left(P \backslash\left\{0_{i}\right\}\right)$, there are $f, g \in B$ with $f(j)=g(j)$ exactly for $j \neq i, 0 \leq j<n$. If $\{f(i), g(i)\} \cap U \neq \emptyset$, say $f(i)=r \in U$, then for $f^{\prime}=F_{r}(f)$ and $g^{\prime}=F_{r}(g)$, we have $\left\{f^{\prime}, g^{\prime}\right\} \subseteq B \cap D^{n},\left(f^{\prime}(i), g^{\prime}(i)\right)=(2,1)$, and $f^{\prime}(j)=g^{\prime}(j)$ for all $j \neq i$. Then put $f^{\prime \prime}=m\left(f^{\prime}, g^{\prime}, \overline{1}\right)$ and notice that $f^{\prime \prime} \in D^{n} \cap B$ is the function $[1,2]_{i}$ that takes value 2 at $i$ and 1 elsewhere. On the other hand, if $\{f(i), g(i)\} \subseteq D$ then put $f^{\prime \prime}=m(f \wedge \overline{2}, g \wedge \overline{2}, \overline{1})$, and again $f^{\prime \prime}=[1,2]_{i} \in D^{n} \cap B$. We have shown that $B$ contains all the functions $[1,2]_{i}, 0 \leq i<n$. Now if $f, g \in D^{n}$ and $f \wedge g=\overline{1}$, then $m(\overline{1}, f, g)=h$ is $f \vee g$-i.e., $h(i)=2$ iff $f(i)=2$ or $g(i)=2$ for all $0 \leq i<n$. It should now be clear that $D^{n} \subseteq B$.

Claim 2. If $\eta=0_{i} \in P$, then $\theta \not \leq \eta$ iff $\left(\overline{1},[1,2]_{i}\right) \in \theta$ and $\left(\overline{2},[2,1]_{i}\right)$ $\in \theta$.

Indeed, suppose that $\theta \not \leq 0_{i}$. Choose $(f, g) \in \theta$ with $f(i) \neq g(i)$. Then as above, either by replacing $(f, g)$ by $\left(F_{r}(f), F_{r}(g)\right)$ for some $r \in U \cap$ $\{f(i), g(i)\}$, or by replacing $(f, g)$ by $(f \wedge \overline{2}, g \wedge \overline{2})$, we get $\left(f^{\prime}, g^{\prime}\right) \in \theta \cap$ $\left(D^{n} \times D^{n}\right), f^{\prime}(i) \neq g^{\prime}(i)$. Now $\left\{f^{\prime} \wedge[1,2]_{i}, g^{\prime} \wedge[1,2]_{i}\right\}=\left\{\overline{1},[1,2]_{i}\right\} ;$ and so we have $\left(\overline{1},[1,2]_{i}\right) \in \theta$. Then also

$$
\left(\left(\overline{2},[2,1]_{i}\right)=\left(m(\overline{1}, \overline{2}, \overline{1}), m\left(\overline{1}, \overline{2},[1,2]_{i}\right)\right) \in \theta,\right.
$$

as required.

Claim 3. $\theta^{\prime} \cap\left(D^{n} \times D^{n}\right) \subseteq \theta$.

To prove this, let

$$
S=\{0, \ldots, n-1\} \backslash T=\left\{i_{0}, \ldots, i_{k-1}\right\}, \quad|S|=k .
$$

Then $\left(\overline{2},[2,1]_{S}\right) \in \theta$. This is clear if $k=0$, for then $[2,1]_{S}=\overline{2}$. It is also clear if $k=1$. If $k>1$ then

$$
\begin{aligned}
{[2,1]_{S} } & =m\left(m\left(\cdots m\left([2,1]_{i_{0}}, \overline{2},[2,1]_{i_{1}}\right), \ldots,\right), \overline{2},[2,1]_{i_{k-1}}\right) \\
& \equiv m(m(\overline{2}, \overline{2}, \overline{2}), \ldots,), \overline{2}, \overline{2}) \quad(\bmod \theta) \\
& =\overline{2}
\end{aligned}
$$

Now let $(f, g) \in \theta^{\prime},\{f, g\} \subseteq D^{n}$. Since $f$ and $g$ agree on $T$, we have $f \wedge[2,1]_{S}=g \wedge[2,1]_{S}$. Also, $f=f \wedge \overline{2}$ and $g=g \wedge \overline{2}$. From $\left([2,1]_{S}, \overline{2}\right) \in \theta$, we conclude that $(f, g) \in \theta$.

Finally, to finish the proof that $\theta=\theta^{\prime}$, suppose that $(f, g) \in \theta^{\prime} \backslash \theta$. Since $\theta \in \mathrm{Con}_{\mathcal{K}} \mathbf{B}$ by assumption, there is a homomorphism $\pi: \mathbf{B} \rightarrow \mathbf{A}$ with $\pi(f) \neq \pi(g)$ and $\theta \subseteq \operatorname{ker}(\pi)$. Let $r \in U$ be arbitrary. Now $\left(F_{r}(f), F_{r}(g)\right) \in$ $\theta^{\prime} \cap\left(D^{n} \times D^{n}\right)$, hence by Claim $3,\left(F_{r}(f), F_{r}(g)\right) \in \theta$ and so $\pi\left(F_{r}(f)\right)$ $=\pi\left(F_{r}(g)\right)$. This means that $F_{r}(\pi(f))=F_{r}(\pi(g))$ for all $r \in U$; and that implies that $\{\pi(f), \pi(g)\} \subseteq D$ since $\pi(f) \neq \pi(g)$. Similarly, we have 
$(f \wedge \overline{2}, g \wedge \overline{2}) \in \theta$ and $\pi(f) \wedge \overline{2}=\pi(g) \wedge \overline{2}$. Since $\{\pi(f), \pi(g)\} \subseteq D$, we obtain

$$
\pi(f)=\pi(f) \wedge \overline{2}=\pi(g) \wedge \overline{2}=\pi(g) .
$$

This contradiction completes our proof that $\theta=\theta^{\prime}$.

ExAmple 13. This is an example, due to R. Willard, of a finitely generated quasivariety $\mathcal{K}$ with $\mathrm{RCM}$ and such that $\mathrm{H}(\mathcal{K})$ is $\mathrm{CM}$ but is not residually small. We define a finite algebra $\mathbf{A}$ with universe $A:=\mathbb{Z}_{4} \times \mathbb{Z}_{2}$ as follows. First define $\lambda_{i}: \mathbb{Z}_{4} \rightarrow \mathbb{Z}_{2}$ for $i<2$ by

\begin{tabular}{c|cc}
$x$ & $\lambda_{0}(x)$ & $\lambda_{1}(x)$ \\
\hline 0 & 0 & 0 \\
1 & 0 & 1 \\
2 & 1 & 0 \\
3 & 1 & 1
\end{tabular}

Then define

$$
\begin{aligned}
f_{i}(\langle x, r\rangle) & =\left\langle 0, \lambda_{i}(x)\right\rangle, \quad i=0,1, \\
f_{2}(\langle x, r\rangle) & =\langle 0, r\rangle, \\
\langle x, r\rangle+\langle y, s\rangle & =\langle x+y, r+s\rangle, \\
\langle x, r\rangle \cdot\langle y, s\rangle & =\langle 2 x y, r s\rangle .
\end{aligned}
$$

Observe that $\langle A ;+, \cdot\rangle$ is a ring (without identity) isomorphic to $2 \mathbb{Z}_{8} \times \mathbb{Z}_{2}$. Let $A_{0}=\{0\} \times \mathbb{Z}_{2} \subseteq A$ and write $\mathbf{0}$ for $\langle 0,0\rangle$ and $\mathbf{1}$ for $\langle 0,1\rangle$. Then we can write $A_{0}=\{\mathbf{0}, \mathbf{1}\}=\mathbf{2}$, which is convenient since $A_{0}$ is closed under,$+ \cdot$ and with respect to these operations is a 2 -element Boolean subring of $\langle A ;+, \cdot\rangle$. We define

$$
\mathbf{A}=\left\langle A ;+, \cdot, \underline{\mathbf{0}}, \underline{\mathbf{1}}, f_{i}(i<3)\right\rangle
$$

and $\mathcal{K}=\mathrm{SP}(\mathbf{A})$.

Clearly, $\mathrm{H}(\mathcal{K})$ is congruence modular. We shall show that $\mathcal{K}$ has WEP and $\mathrm{H}(\mathcal{K})$ is residually large. We first show that Con $\mathbf{A}$ is a 4-element chain with congruences $0_{A}<\mu<\theta<1_{A}$ where

$$
\mu=0_{\mathbb{Z}_{4}} \times 1_{\mathbb{Z}_{2}}, \quad \theta=\gamma \times 1_{\mathbb{Z}_{2}},
$$

and $\gamma$ is $\{0,2\}^{2} \cup\{1,3\}^{2}$.

First check that $\mu$ is a congruence. Next observe that the presence of + implies that $0_{A} \prec \mu$. Finally, the presence of $f_{0}, f_{1}$ guarantees that if $a, b \in A$ with $a \neq b$ then $\operatorname{Cg}_{\mathbf{A}}(a, b) \cap \mu \neq 0_{A}$, proving $\mu$ is the monolith of $\mathbf{A}$. Now $\mathbf{A} / \mu$ is term-equivalent to the four-element ring $2 \mathbb{Z}_{8}$, whose congruences are known, completing the picture of Con $\mathbf{A}$.

Since the ring $2 \mathbb{Z}_{8}$ generates a residually large variety, so does $\mathbf{A} / \mu$. Thus $\mathrm{H}(\mathcal{K})$ is residually large. 
It remains to prove WEP. It will suffice to show that each finite $\mathbf{B} \in \mathcal{K}$ has WEP. Choose finite $\mathbf{B} \in \mathcal{K}$ and represent it as $\mathbf{B} \leq \mathbf{A}^{n}$ with $n$ minimum. For each $i<n$ let $\mu_{i}^{\prime}=\left.\left(0_{A} \times \cdots \times 0_{A} \times \mu \times 0_{A} \times \cdots \times 0_{A}\right)\right|_{B}$ where the lone copy of $\mu$ occurs in the $i$ th coordinate. Similarly, let as usual $0_{i}=\left(1_{A} \times \cdots \times\right.$ $\left.1_{A} \times 0_{A} \times 1_{A} \times \cdots \times 1_{A}\right)\left.\right|_{B}$ and $0_{i}^{\prime}=\left.\left(0_{A} \times \cdots \times 0_{A} \times 1_{A} \times 0_{A} \times \cdots \times 0_{A}\right)\right|_{B}$. Then $\mu_{i}^{\prime}, 0_{i}, 0_{i}^{\prime} \in \mathbf{C o n} \mathbf{B}$.

Put $B_{0}=B \cap\left(A_{0}\right)^{n}$. Observe that $f_{i}(B) \subseteq B_{0}$ for $i<3$, and that $\left\langle B_{0} ;+, \cdot, \underline{\mathbf{0}}, \underline{\mathbf{1}}\right\rangle$ is a Boolean subring of $\mathbf{2}^{n}$. For each $i<n$ let

$$
e_{i}=(\mathbf{0}, \ldots, \mathbf{1}, \ldots, \mathbf{0}) \in \mathbf{2}^{n}
$$

with the single $\mathbf{1}$ occurring in the $i$ th coordinate.

Claim 1. $e_{i} \in B_{0}$ for each $i<n$.

To prove the claim, fix $i<n$. By minimality of $n$ we have $a, b \in B$ with $\llbracket a \neq b \rrbracket=\{i\}$. There exists $j<3$ such that $f_{j}\left(a_{i}\right) \neq f_{j}\left(b_{i}\right)$, so by replacing $a, b$ with $a^{\prime}:=f_{j}(a), b^{\prime}:=f_{j}(b)$ we may assume that $a, b \in B_{0}$. Then $e_{i}=a+b \in B$ as required. Since $B_{0}$ is a Boolean subring of $\mathbf{2}^{n}$, it follows that $B_{0}=\mathbf{2}^{n}$.

Claim 2. If $i<n$ and $c, d \in B$ and $c_{i} \neq d_{i}$, then $\mu_{i}^{\prime} \leq \operatorname{Cg}_{\mathbf{B}}(c, d)$.

As in the proof of Claim 1, we have a unary term operation $f(x)$ such that $f(B) \subseteq B_{0}$ and $\left\{f(c)_{i}, f(d)_{i}\right\}=\{\mathbf{0}, \mathbf{1}\}$. The polynomial $x \mapsto x \cdot e_{i}$ maps $\{f(c), f(d)\}$ to $\left\{\underline{\mathbf{0}}, e_{i}\right\}$, proving $\left(\underline{\mathbf{0}}, e_{i}\right) \in \mathrm{Cg}_{\mathbf{B}}(c, d)$. Then for every $a \in B$, $\left(a, a+e_{i}\right)=\left(a+\underline{\mathbf{0}}, a+e_{i}\right) \in \operatorname{Cg}_{\mathbf{A}}(c, d)$, proving $\mu_{i}^{\prime} \leq \operatorname{Cg}_{\mathbf{A}}(c, d)$.

Now to prove that $\mathbf{B}$ has WEP, we use Lemma 14 from the next section. Let $\alpha$ be a minimal congruence of $\mathbf{B}$ and let $\beta \in$ Con $\mathbf{B}$ be maximal with respect to satisfying $\alpha \wedge \beta=0_{B}$. Then by Claim 2, $\alpha=\mu_{i}^{\prime}$ for some $i<n$ and $\beta \leq 0_{i}$. Since $\mu_{i}^{\prime} \wedge 0_{i}=0_{B}$ we have $0_{i} \leq \beta$. Therefore $\beta=0_{i}$, so $\beta \in \operatorname{Con}_{\mathcal{K}}(\mathbf{B})$ as required.

Notice that since every subalgebra $\mathbf{B}$ of $\mathbf{A}$ is subdirectly irreducible with non-Abelian monolith $\mathrm{Cg}_{\mathbf{B}}(\mathbf{0}, \mathbf{1})$, it follows by Theorem 2.3 and 4.1 of M. Maróti and R. McKenzie [17] that $\mathcal{K}$ has Willard terms. Hence, by Theorem $1(4), \mathcal{K}$ is $\operatorname{RSD}(\wedge)$ and thus, by Theorem $7, \mathcal{K}$ is finitely axiomatizable.

6. A characterization of finite $\mathcal{F}$ for which $\operatorname{SP}(\mathcal{F}) \models \operatorname{RSD}(\wedge)$. Let $\mathcal{F}$ be any finite set of finite algebras of the same signature. According to Theorem 4.1 in M. Maróti and R. McKenzie [17], SP $(\mathcal{F})$ satisfies PCC if and only if every minimal congruence of every algebra in $\mathrm{S}(\mathcal{F})$ is non-Abelian. Our characterization of those $\mathcal{F}$ for which $\operatorname{SP}(\mathcal{F}) \mid=\operatorname{RSD}(\wedge)$, equivalently, $\mathrm{SP}(\mathcal{F}) \models \mathrm{PCC}+\mathrm{WEP}$, is a little more complicated. If a quasivariety $\mathcal{K}$ satisfies PCC then there are several ways to characterize WEP for $\mathcal{K}$. We 
use $\alpha^{c}$ to denote the pseudo-complement of a congruence $\alpha$ on some algebra in $\mathcal{K}$. Supposing that $\mathcal{K}=\mathrm{PCC}$, these statements are equivalent to WEP for $\mathcal{K}$ :

- For $\mathbf{A} \in \mathcal{K}$ and $\alpha, \beta \in$ Con $\mathbf{A}$ with $\alpha \wedge \beta=0_{A}, \bar{\alpha}^{\mathcal{K}} \wedge \bar{\beta}^{\mathcal{K}}=0_{A}$.

- For $\mathbf{A} \in \mathcal{K}$ and $\alpha, \beta \in$ Con $\mathbf{A}$ with $\alpha \wedge \beta=0_{A}, \alpha \wedge \bar{\beta}^{\mathcal{K}}=0_{A}$.

- For $\mathbf{A} \in \mathcal{K}$ and $\alpha \in \operatorname{Con} \mathbf{A}, \alpha^{c} \in \operatorname{Con}_{\mathcal{K}} \mathbf{A}$.

If $\mathcal{K}$ is locally finite but does not necessarily satisfy $\mathrm{PCC}$, there is another way to characterize WEP.

Lemma 14. Let $\mathcal{K}$ be a locally finite quasivariety.

(1) $\mathcal{K}$ satisfies WEP iff for every finite $\mathbf{A} \in \mathcal{K}$ and atom $\alpha \in$ Con $\mathbf{A}$, all maximal members of the set $\{\beta \in$ Con $\mathbf{A}: \beta \nsupseteq \alpha\}$ belong to $\operatorname{Con}_{\mathcal{K}} \mathbf{A}$.

(2) If $\mathcal{K}$ satisfies $\mathrm{PCC}$ then $\mathcal{K}$ satisfies WEP iff for every finite $\mathbf{A} \in$ $\mathcal{K}$ and atom $\alpha \in \mathrm{Con} \mathbf{A}$, the pseudo-complement of $\alpha$ belongs to $\mathrm{Con}_{\mathcal{K}} \mathbf{A}$.

Proof. Suppose that $\alpha$ is an atom in Con $\mathbf{A}$ and $\beta \nsupseteq \alpha$ while for every congruence $\gamma>\beta$, we have $\gamma \geq \alpha$. Then $\beta$ is strictly meet irreducible and its unique cover is $\beta \vee \alpha$. If $\mathcal{K}$ has the weak extension property, then $\bar{\beta}^{\mathcal{K}} \wedge \bar{\alpha}^{\mathcal{K}}=0_{A}$, implying that $\bar{\beta}^{\mathcal{K}}=\beta$, so $\beta \in \operatorname{Con}_{\mathcal{K}} \mathbf{A}$. This shows the necessity of the condition in (1). To see that this condition is also sufficient, suppose that $\mathcal{K}$ does not have the weak extension property. Then in fact there is some finite $\mathbf{A} \in \mathcal{K}$ and congruences $\alpha, \beta$ such that $\alpha \wedge \beta=0_{A}$ and $\alpha \wedge \bar{\beta}^{\mathcal{K}}>0_{A}$. Let $\alpha_{0}$ be any atom (minimal congruence) below $\alpha \wedge \bar{\beta}^{\mathcal{K}}$. Now $\alpha_{0} \wedge \beta=0_{A}$. Let $\beta^{\prime}$ be any maximal member of the set of congruences $\tau$ with $\alpha_{0} \not \leq \tau$ and $\beta \leq \tau$. We have to see that $\beta^{\prime} \notin \operatorname{Con}_{\mathcal{K}} \mathbf{A}$. But if $\beta^{\prime} \in \operatorname{Con}_{\mathcal{K}} \mathbf{A}$, then $\bar{\beta}^{\mathcal{K}} \leq \beta^{\prime}$, implying that $\bar{\beta}^{\mathcal{K}} \nsucceq \alpha_{0}$, which is not the case. This completes our argument for (1).

Now assume that $\mathcal{K}$ satisfies PCC. Then if $\mathbf{A} \in \mathcal{K}$ and $\alpha$ is an atom in Con $\mathbf{A}$, there is a unique maximal member of the set of congruences not dominating $\alpha$, namely $\alpha^{c}$. Thus (2) can be seen as a specialization of (1) to this case.

If $\mathcal{K}$ is any quasivariety and if $\alpha$ is an atom in the congruence lattice of an algebra $\mathbf{A} \in \mathcal{K}$, and if the pseudo-complement $\alpha^{c}$ of $\alpha$ exists, then $\mathbf{A} / \alpha^{c}$ is a subdirectly irreducible algebra. Unless this subdirectly irreducible algebra belongs to $\mathcal{K}$, the quasivariety $\mathcal{K}$ cannot have WEP. These observations imply, for instance, that if $\mathcal{K}$ is locally finite and satisfies $\operatorname{RSD}(\wedge)$ then it is generated by its finite subdirectly irreducible members.

Recall that when $\alpha$ is a non-Abelian atom in the congruence lattice of a finite algebra, the pseudo-complement of $\alpha$ does exist. (See D. Hobby and R. McKenzie [11].) 
Lemma 15. Assume that $\mathcal{K}$ is a quasivariety, $\mathcal{K} \models \mathrm{PCC},\left\{\mathbf{A}_{0}, \ldots, \mathbf{A}_{k}\right\}$ is a finite collection of finite algebras in $\mathcal{K}$, and $\mathbf{A} \leq \prod_{i} \mathbf{A}_{i}$ is a subdirect product. Let $\alpha$ be an atom in $\mathbf{C o n} \mathbf{A}$ and $\beta=\alpha^{c}$. Choose $i, 0 \leq i \leq k$, such that $\alpha \not \leq 0_{i}$. Then

(i) $0_{i} \leq \beta$;

(ii) if $\left|\mathbf{A}_{i}\right| \leq n$ and the cardinality of the free algebra on $n$ generators in $\mathcal{K}$ is $m$, then there are $\mathbf{B}_{0}, \ldots, \mathbf{B}_{m-1} \in \mathbf{S}\left(\left\{\mathbf{A}_{0}, \ldots, \mathbf{A}_{k}\right\}\right)$ with $\mathbf{B}_{0}=\mathbf{A}_{i}$, and there is a subdirect product $\mathbf{B} \leq \prod_{j} \mathbf{B}_{j}$, and an atom $\alpha^{\prime}$ in $\mathbf{C o n} \mathbf{B}$, such that for $\beta^{\prime}=\alpha^{\prime c}$, we have $\beta^{\prime} \geq 0$ and $\mathbf{A} / \beta \cong$ $\mathbf{B} / \beta^{\prime} \in \mathrm{H}\left(\mathbf{B}_{0}\right)=\mathrm{H}\left(\mathbf{A}_{i}\right)$.

Proof. Clearly, since $\alpha \not \leq 0_{i}$ we have $\alpha \wedge 0_{i}=0_{A}$, implying that $0_{i} \leq$ $\alpha^{c}=\beta$. Choose a subset $\left\{a_{0}, \ldots, a_{n-1}\right\} \subseteq A$ which intersects all $0_{i}$-classes and such that $a_{0} \neq a_{1}$ and $\left(a_{0}, a_{1}\right) \in \alpha$. Let $\mathbf{B}$ be the subalgebra of $\mathbf{A}$ generated by $\left\{a_{0}, \ldots, a_{n-1}\right\}$. Thus $|\mathbf{B}| \leq m$. Note that $\left(a_{0}, a_{1}\right) \notin 0_{i}$, since otherwise $\alpha=\operatorname{Cg}_{\mathbf{B}}\left(a_{0}, a_{1}\right) \leq 0_{i}$. It is then easy to see that there are $j_{0}=$ $i, j_{1}, \ldots, j_{m-1} \in\{0, \ldots, k\}$ such that $\left.\left(\bigwedge_{0<r<m} 0_{j_{r}}\right)\right|_{\mathbf{B}}=0_{B}$. Let $\beta^{\prime}=\left.\beta\right|_{\mathbf{B}}$ and $\alpha^{\prime \prime}=\left.\alpha\right|_{\mathbf{B}}$. Clearly, $\alpha^{\prime \prime} \wedge \beta^{\prime}=0_{B}, \alpha^{\prime \prime}>0_{B}$, and since $\mathbf{B}$ projects onto $\mathbf{A}_{i}=\mathbf{A}_{j_{0}}$, every congruence strictly larger than $\beta^{\prime}$ contains $\alpha^{\prime \prime}$. Thus $\beta^{\prime}$ is the pseudo-complement of $\alpha^{\prime \prime}$ in Con $\mathbf{B}$. Let $\alpha^{\prime}$ be any atom of Con $\mathbf{B}$ lying below $\alpha^{\prime \prime}$. Then $\beta^{\prime}=\alpha^{\prime c}$ in Con $\mathbf{B}$. Now let $\mathbf{B}_{r} \subseteq \mathbf{A}_{j_{r}}$ be the projection of $\mathbf{B}$ at the $j_{r}$ coordinate. Thus $\mathbf{B}_{0}=\mathbf{A}_{i}$. Replacing $\mathbf{B}$ by the subdirect product of $\mathbf{B}_{0}, \ldots, \mathbf{B}_{m-1}$ canonically isomorphic to it, and $\beta^{\prime}, \alpha^{\prime}$ by the corresponding congruences, we have the desired conclusions.

TheOREM 16. Suppose that $\mathcal{K}=\operatorname{SP}(\mathcal{F})$ where $\mathcal{F}$ is a finite collection of finite algebras and $\mathbf{S}(\mathcal{F})=\mathcal{F}$. Let $n=\max (|\mathbf{A}|: \mathbf{A} \in \mathcal{F})$ and let $f$ be the cardinality of the free algebra on $n$ generators in $\mathcal{K}$. Then $\mathcal{K} \models \operatorname{RSD}(\wedge)$ iff the following are true.

(i) Every atom in the congruence lattice of any member of $\mathcal{F}$ is nonAbelian.

(ii) Let $\mathbf{A} \leq \prod_{0 \leq i<f} \mathbf{A}_{i}$ be a subdirect product with $\left\{\mathbf{A}_{0}, \ldots, \mathbf{A}_{f-1}\right\} \subseteq \mathcal{F}$. Let $\alpha$ be any atom in $\operatorname{Con} \mathbf{A}$ and $\beta=\alpha^{c}$. Then $\mathbf{A} / \beta$ is isomorphic to a member of $\mathcal{F}$.

Proof. The necessity of (i) follows from M. Maróti and R. McKenzie [17, Theorem 4.1]. To see that (ii) is necessary, observe that by Lemma $14, \mathbf{A} / \beta$ must belong to $\mathcal{K}$; but it is a subdirectly irreducible algebra, and therefore must actually be isomorphic to a subalgebra of some member of $\mathcal{F}$.

Now suppose that (i) and (ii) hold. Then $\mathcal{K} \models$ PCC. To establish that $\mathcal{K} \models$ WEP, using Lemma 14(2), let $\mathbf{A} \leq \prod_{0<i<k} \mathbf{A}_{i}$ be a subdirect product, with $k$ a non-negative integer and $\mathbf{A}_{i} \in \mathcal{F}$. Let $\alpha$ be an atom in Con $\mathbf{A}$ 
and $\beta=\alpha^{c}$. Now it follows from Lemma 15, in conjunction with (ii), that $\mathbf{A} / \beta \in \mathcal{K}$.

It is obvious that the above theorem supplies an algorithm to effectively recognize whether $\operatorname{SP}(\mathcal{F})$ is $\operatorname{RSD}(\wedge)$. It is not obvious if there may be some algorithm to solve this problem which lies low in the complexity hierarchy.

To conclude, we present three related problems that seem to us very interesting and difficult.

Problem 17. Suppose that $\mathcal{K}$ is a finitely generated quasivariety satisfying $\mathrm{CM}+\mathrm{RCM}$. Is it finitely axiomatizable?

Problem 18. Suppose that $\mathcal{K}$ is a finitely generated quasivariety satisfying RCM. Is it finitely axiomatizable?

Problem 19. Suppose that $\mathcal{K}$ is a finitely generated quasivariety satisfying WEP. Is it finitely axiomatizable?

Acknowledgements. While working on this paper, the second author (Maróti) was supported by the Hungarian National Foundation for Scientific Research (OTKA), grant nos. T48809 and T60148, the third author (McKenzie) was supported by US NSF grant DMS-0245622, and the fourth author (Nurakunov) was supported by a COBASE grant awarded by the National Academies of the US.

\section{References}

[1] K. Baker, Primitive satisfaction and equational problems for lattices and other algebras, Trans. Amer. Math. Soc. 190 (1974), 125-150.

[2] - Finite equational bases for finite algebras in a congruence distributive equational class, Adv. Math. 24 (1977), 207-243.

[3] V. P. Belkin, Quasi-identities of finite rings and lattices, Algebra i Logika 17 (1978), 247-259 (in Russian).

[4] G. Birkhoff, On the structure of abstract algebras, Proc. Cambridge Philos. Soc. 31 (1935), 433-454.

[5] W. J. Blok and D. Pigozzi, A finite basis theorem for quasivarieties, Algebra Universalis 22 (1986), 1-13.

[6] J. Czelakowski and W. Dziobiak, Congruence distributive quasivarieties whose finitely subdirectly irreducible members form a universal class, ibid. 27 (1990), 128149.

[7] W. Dziobiak, Finitely generated congruence distributive quasivarieties of algebras, Fund. Math. 133 (1989), 47-57.

[8] -, Quasivarieties of Sugihara semilattices with involution, Algebra and Logic 39 (2000), 26-36.

[9] R. Freese and R. McKenzie, Residually small varieties with modular congruence lattices, Trans. Amer. Math. Soc. 264 (1981), 419-430.

[10] G. Grätzer and H. Lakser, A note on the implicational class generated by a class of structures, Canad. Math. Bull. 16 (1973), 603-605. 
[11] D. Hobby and R. McKenzie, The Structure of Finite Algebras, Contemp. Math. 76, Amer. Math. Soc., 1988.

[12] J. Ježek, M. Maróti and R. McKenzie, Quasi-equational theories of flat algebras, Czechoslovak Math. J. 55 (2005), 665-675.

[13] K. Kearnes and R. McKenzie, Commutator theory for relatively modular quasivarieties, Trans. Amer. Math. Soc. 331 (1992), 465-502.

[14] R. Lyndon, Identities in two-valued calculi, ibid. 71 (1951), 457-465.

[15] —, Identities in finite algebras, Proc. Amer. Math. Soc. 5 (1954), 8-9.

[16] A. I. Maltsev, Several remarks on quasivarieties of algebraic systems, Algebra i Logika 5 (1966), 3-9 (in Russian).

[17] M. Maróti and R. McKenzie, Finite basis problems and results for quasivarieties, Studia Logica 78 (2004), 293-320.

[18] R. McKenzie, Finite forbidden lattices, in: Universal Algebra and Lattice Theory (Puebla, 1982), Lecture Notes in Math. 1004, Springer, Berlin, 1983, 176-205.

[19] - Finite equational bases for congruence modular varieties, Algebra Universalis 24 (1987), 224-250.

[20] - , Tarski's finite basis problem is undecidable, Int. J. Algebra Comput. 6 (1996), 49-104.

[21] —, A compatible flat algebra without finite quasi-equational basis, preprint.

[22] R. McKenzie, G. McNulty and W. Taylor, Algebras, Lattices, Varieties, Vol. I, Wadsworth \& Brooks/Cole Advanced Books and Software, Monterey, CA, 1987.

[23] A. Nurakunov, Characterization of relatively distributive quasivarieties of algebras, Algebra and Logic 29 (1990), 451-458.

[24] -, Quasi-identities of congruence-distributive quasivarieties of algebras, Siberian Math. J. 42 (2001), 108-118.

[25] D. Pigozzi, Finite basis theorems for relatively congruence distributive quasivarieties, Trans. Amer. Math. Soc. 310 (1988), 499-533.

[26] R. Willard, A finite basis theorem for residually finite, congruence meet-semidistributive varieties, J. Symbolic Logic 65 (2000), 187-200.

[27] —, Extending Baker's theorem, Algebra Universalis 45 (2001), 335-344.

Department of Mathematics

University of Puerto Rico

Mayagüez Campus

Mayagüez, PR 00681-9018, U.S.A.

E-mail: w.dziobiak@gmail.com

Department of Mathematics

Vanderbilt University

Nashville, TN 37235, U.S.A.

E-mail: ralph.n.mckenzie@vanderbilt.edu
Bolyai Institute University of Szeged H-6720 Szeged, Hungary E-mail: mmaroti@math.u-szeged.hu

Institute of Mathematics National Academy of Science Chui pr., 265a Bishkek, 720071, Kyrghyz Republic

E-mail: anvar@aknet.kg

Received 29 November 2006;

in revised form 7 November 2008 